\title{
THE BRAUER-MANIN PAIRING, CLASS FIELD THEORY, AND MOTIVIC HOMOLOGY
}

\author{
TAKAO YAMAZAKI
}

\begin{abstract}
For a smooth proper variety over a $p$-adic field, its Brauer group and abelian fundamental group are related to higher Chow groups by the BrauerManin pairing and class field theory. We generalize this relation to smooth (possibly nonproper) varieties, using motivic homology and a variant of Wiesend's ideal class group. Several examples are discussed.
\end{abstract}

\section{$\S 1$. Introduction}

\subsection{The Brauer-Manin pairing and class field theory}

Let $p$ be a prime number, and let $k$ be a finite extension of $\mathbb{Q}_{p}$. Let $X$ be a smooth variety over $k$. For $i \in \mathbb{Z}_{\geq 0}$, we write $X_{(i)}$ for the set of all points of $X$ of dimension $i$. Let $\operatorname{Br}(X)$ be the cohomological Brauer group of $X$, and let $\pi_{1}^{\mathrm{ab}}(X)$ be the abelian étale fundamental group of $X$. For any $x \in X_{(0)}$, local class field theory yields canonical maps

$$
\psi_{x}^{*}: \operatorname{Br}(x) \cong \mathbb{Q} / \mathbb{Z}, \rho_{x}: k(x)^{*} \rightarrow \pi_{1}^{\mathrm{ab}}(x) .
$$

As for the first map, it is convenient for our purpose to consider its dual. Putting $A^{*}:=\operatorname{Hom}(A, \mathbb{Q} / \mathbb{Z})$ for an abelian group $A$, we define $\psi_{x}$ to be the composition of the dual of $\psi_{x}^{*}$ and the canonical inclusion

$$
\psi_{x}: \mathbb{Z} \hookrightarrow \hat{\mathbb{Z}} \cong \operatorname{Br}(x)^{*}
$$

Since both of $\operatorname{Br}(-)^{*}$ and $\pi_{1}^{\mathrm{ab}}(-)$ are covariant functorial, we get homomorphisms

Received November 13, 2011. Revised February 24, 2012. Accepted March 21, 2012. 2010 Mathematics Subject Classification. Primary 11S25; Secondary 14G20, 14C25.

The author's work partially supported by Japan Society for the Promotion of Science Grant-in-Aid for Young Scientists (A) (22684001).

(C) 2013 by The Editorial Board of the Nagoya Mathematical Journal 


$$
\begin{aligned}
& \tilde{\psi}_{X}: Z_{0}(X):=\bigoplus_{x \in X_{(0)}} \mathbb{Z} \rightarrow \operatorname{Br}(X)^{*}, \\
& \tilde{\rho}_{X}: Z_{0}^{1}(X):=\bigoplus_{x \in X_{(0)}} k(x)^{*} \rightarrow \pi_{1}^{\mathrm{ab}}(X)
\end{aligned}
$$

by taking the direct sum of the $\psi_{x}$ and $\rho_{x}$ values.

When $X$ is proper over $k$, Manin [23], Bloch [1], and Saito [29] observed that $\tilde{\psi}_{X}$ and $\tilde{\rho}_{X}$ factor, respectively, through

$$
\begin{aligned}
& \mathrm{CH}_{0}(X):=\operatorname{coker}\left[\bigoplus_{y \in X_{(1)}} k(y)^{*} \rightarrow Z_{0}(X)\right], \\
& S K_{1}(X):=\operatorname{coker}\left[\bigoplus_{y \in X_{(1)}} K_{2} k(y) \rightarrow Z_{0}^{1}(X)\right]
\end{aligned}
$$

the induced pairing $\mathrm{CH}_{0}(X) \times \operatorname{Br}(X) \rightarrow \mathbb{Q} / \mathbb{Z}$ and the induced map $S K_{1}(X) \rightarrow \pi_{1}^{\mathrm{ab}}(X)$ are called the Brauer-Manin pairing and reciprocity map of class field theory, respectively. Both are studied intensively by several authors (for the Brauer-Manin pairing, see [4]-[6], [28], [31], and [46]; for the reciprocity map, see [18], [19], [32], [40], [41], and [47]).

If $X$ is not proper over $k$, however, $\tilde{\psi}_{X}$ and $\tilde{\rho}_{X}$ do not factor through $\mathrm{CH}_{0}(X)$ or $S K_{1}(X)$. To this end, we will introduce good quotients of $Z_{0}(X)$ and $Z_{0}^{1}(X)$.

\subsection{Wiesend's tame ideal class group}

Let $V$ be a variety over a field $F$. Take $y \in V_{(1)}$. Let $C(y)$ be the closure of $\{y\}$ in $V$, let $\tilde{C}(y) \rightarrow C(y)$ be the normalization, let $\tilde{C}(y) \hookrightarrow \bar{C}(y)$ be the smooth completion, and let $C_{\infty}(y)=\bar{C}(y) \backslash \tilde{C}(y)$. For $x \in C_{\infty}(y)$, we take a uniformizer $\pi_{x} \in F(y)^{*}$ at $x$. We define for $r \in \mathbb{Z}_{>0}$

$$
U K_{r}^{M} F(y):=\operatorname{ker}\left[K_{r}^{M} F(y) \rightarrow \bigoplus_{x \in C_{\infty}(y)}\left(K_{r-1}^{M} F(x) \oplus K_{r}^{M} F(x)\right)\right]
$$

Here the $x$-component of the map is defined by $a \mapsto\left(\partial_{x}(a), \partial_{x}\left(\left\{\pi_{x}\right\} \cup a\right)\right)$ for $a \in K_{r}^{M} F(y)$, where $\partial_{x}$ is the tame symbol at $x$. This group does not depend on the choice of $\pi_{x}$. If $V$ is proper over $F$, then $U K_{r}^{M} F(y)=K_{r}^{M} F(y)$. For example, $U K_{1}^{M} F(y)$ is the group of rational functions on $\bar{C}(y)$ which takes value 1 at all points of $C_{\infty}(y)$. The following definition is a natural outcome of an idea of Wiesend [45]. 
Definition 1.1. Let $V$ be a variety over a field $F$, and let $r \in \mathbb{Z}_{\geq 0}$. We define Wiesend's tame ideal class group of degree $r$ to be

$$
C_{r}(V):=\operatorname{coker}\left[\bigoplus_{y \in V_{(1)}} U K_{r+1}^{M} F(y) \hookrightarrow \bigoplus_{y \in V_{(1)}} K_{r+1}^{M} F(y) \stackrel{(*)}{\rightarrow} \bigoplus_{x \in V_{(0)}} K_{r}^{M} F(x)\right]
$$

where $(*)$ is the boundary map of Gersten complex of the Milnor $K$-sheaf. (In particular, its image is in the direct sum.)

By definition, $C_{0}(V)$ and $C_{1}(V)$ are quotients of $Z_{0}(V)$ and $Z_{0}^{1}(V)$, respectively. If $V$ is proper over $F$, then we have $C_{0}(V)=\mathrm{CH}_{0}(V)$ and $C_{1}(V)=S K_{1}(V)$.

REMARK 1.2. Suppose that $V=\bar{C} \backslash C_{\infty}$, where $\bar{C}$ is a smooth projective geometrically irreducible curve over $F$ and where $C_{\infty}$ is a closed reduced subvariety of $\bar{C}$. Then our definition of $C_{0}(V)$ coincides with that of the group of classes of divisors on $\bar{C}$ prime to $C_{\infty}$ modulo $C_{\infty}$-equivalence defined in [36, Chapitre 5, Section 2]. It is proved in [36, Chapitre 5, Théorème 1] that $\operatorname{ker}\left(\operatorname{deg}: C_{0}(V) \rightarrow \mathbb{Z}\right)$ is represented by a semiabelian variety $J$, which is called the generalized Jacobian variety of $\bar{C}$ with modulus $C_{\infty}$. (Here the degree map deg: $C_{0}(V) \rightarrow \mathbb{Z}$ is induced by the usual degree map $Z_{0}(V) \rightarrow \mathbb{Z}$.) For simplicity, we call $J$ the generalized Jacobian of $V$. One can also interpret $C_{0}(V)$ as the Picard group of the singular curve obtained from $\bar{C}$ by contracting all points of $C_{\infty}$ to one point (see [36, Chapitre 5, Section 2, and Chapitre 4, Section 4]). On the other hand, $C_{0}(V)$ is also isomorphic to the relative Picard group $\operatorname{Pic}\left(\bar{C}, C_{\infty}\right)$ (see $[38$, Theorem 3.1]).

\subsection{Motivic homology}

Let $V$ be a smooth variety over a perfect field $F$, and let $i, j \in \mathbb{Z}$. Its motivic homology $H_{i}^{M}(V, \mathbb{Z}(j))$ is defined to be the group of homomorphisms $\operatorname{Hom}_{\mathrm{DM}_{\mathrm{Nis}}^{\mathrm{eff}},-(F)}(\mathbb{Z}(j)[i], M(V))$ in Voevodsky's category (see [11], [26, (14.17)]). When $j=0$, the group $H_{i}^{M}(V, \mathbb{Z}(0))$ agrees with Suslin's algebraic singular homology $h_{i}(V)$ (see [38]) and admits a (relatively simple) description in terms of algebraic cycles (see [42, (3.2.7)], [26, (14.18)]). There is a similar but complicated description also for $j \neq 0$ (see $[11,(9.4)]$ ). The following theorem, which plays a crucial role in our paper, provides a simpler description in a special case. 
THEOREM 1.3. Let $V$ be a smooth variety over a perfect field $F$, and let $r \in \mathbb{Z}_{\geq 0}$. Then there is a canonical isomorphism

$$
C_{r}(V) \cong H_{-r}^{M}(V, \mathbb{Z}(-r)) .
$$

When $r=0$, using the comparison of $H_{0}^{M}(V, \mathbb{Z}(0))$ with $h_{0}(V)$ recalled above, this follows from a result of Schmidt ([34, Theorem 5.1]). We will prove this theorem by reducing to the case $r=0$ in Section 2 . In what follows, we often identify $C_{r}(V)$ and $H_{-r}^{M}(V, \mathbb{Z}(-r))$.

REMARK 1.4. For a smooth projective variety $V$ over a finite field, unramified class field theory (see [21]) relates $\mathrm{CH}_{0}(V)$ with the abelian fundamental group of $V$. This has been generalized by Schmidt and Spieß [35] to a smooth (possibly nonproper) variety $V$, in which $\mathrm{CH}_{0}(V)$ is replaced by Suslin's algebraic singular homology $h_{0}(V)$. (Note that $h_{0}(V) \cong C_{0}(V)$ by Schmidt's theorem mentioned above. See also recent works of Geisser [12], [13] for a further generalization.) The basic strategy of our paper is to follow their argument over a $p$-adic base field.

\subsection{Open varieties over a $p$-adic field}

We go back to the situation of Section 1.1. Schmidt and Spieß [35] constructed a map connecting motivic homology and étale cohomology with compact support, which we will recall in Section 3. As an application, we deduce the following proposition in Section 4.

Proposition 1.5. Let $X$ be a smooth variety over a finite extension $k$ of $\mathbb{Q}_{p}$. The homomorphisms (1.1.1) induce well-defined homomorphisms

$$
\psi_{X}: C_{0}(X) \rightarrow \operatorname{Br}(X)^{*}, \quad \rho_{X}: C_{1}(X) \rightarrow \pi_{1}^{\mathrm{ab}}(X)
$$

In Section 5, we consider the case where $X$ is a curve. In this case, the map $\psi_{X}$ was already studied by Scheiderer and van Hamel [33] (see Theorem 5.1 below), and the map $\rho_{X}$ is closely related to work of Hiranouchi [16] (see Theorem 5.4 and Remark 5.5 below). When $X$ is of dimension 2 or higher, the maps $\psi_{X}$ and $\rho_{X}$ are not very close to an isomorphism even if $X$ is projective over $k$. We study several examples of surfaces in Section 6. As a sample, here we mention the following result. (We write $\bar{V}=V \times_{k} \bar{k}$ for a variety $V$ over a field $k$ with an algebraic closure $\bar{k}$.)

THEOREM 1.6. Let $X$ be a smooth projective geometrically irreducible surface over a finite extension $k$ of $\mathbb{Q}_{p}$. Suppose that $\bar{X}$ is rational. Let $U$ be an open subvariety of $X$. 
(1) Suppose that the irreducible components of $\bar{X} \backslash \bar{U}$ generate the NéronSeveri group $N S(\bar{X})$ of $\bar{X}$. Then, the kernel of $\psi_{U}$ is the maximal divisible subgroup of $C_{0}(U)$. However, there is an example of $U$ such that $\operatorname{ker}\left(\psi_{U} \otimes \mathbb{Z} / n \mathbb{Z}\right) \neq 0$ for all $n \in \mathbb{Z}_{>0}$ divisible by some (fixed) integer $N$.

(2) The kernel of $\rho_{U}$ is the maximal divisible subgroup of $C_{1}(U)$, and $\rho_{U} \otimes$ $\mathbb{Z} / n \mathbb{Z}$ is bijective for all $n \in \mathbb{Z}_{>0}$.

Note that, if $X$ is a (projective smooth) rational surface, then $\operatorname{ker}\left(\psi_{X}\right)=0$ and $\operatorname{ker}\left(\psi_{X} \otimes \mathbb{Z} / n \mathbb{Z}\right)=0$ for all sufficiently divisible $n$ (see Theorem 6.2). Note also that there are examples of (nonrational) projective smooth surfaces $X$ and $X^{\prime}$ for which $\operatorname{ker}\left(\psi_{X}\right)$ and $\operatorname{ker}\left(\rho_{X^{\prime}}\right)$ are not divisible (see [28], $[32])$.

\subsection{Conventions}

Let $A$ be an abelian group. For a nonzero integer $n$, we write $A[n]$ and $A / n$ for the kernel and cokernel of the map $n: A \rightarrow A$. This notation is sometimes used when $n=\infty$, in which case we mean that $A[n]=A_{\text {Tor }}$ is the subgroup of torsion elements in $A$, and $A / n=A \otimes \mathbb{Q} / \mathbb{Z}$. We write $A_{\mathbb{Q}}$ for $A \otimes_{\mathbb{Z}} \mathbb{Q}$. We define $A_{\text {Div }}:=\operatorname{Im}[\operatorname{Hom}(\mathbb{Q}, A) \rightarrow \operatorname{Hom}(\mathbb{Z}, A)=A]$ to be the maximal divisible subgroup in $A$, and we define $A_{\text {div }}:=\bigcap_{n \in \mathbb{Z}_{>0}} n A$ to be the subgroup of divisible elements in $A$. Note that we always have $A_{\text {Div }} \subset A_{\text {div }}$ and that $A_{\text {Div }}=A_{\text {div }}$ holds if $A[n]$ is finite for all $n \in \mathbb{Z}_{>0}$.

Let $f: A \rightarrow B$ be a homomorphism of abelian groups. We write $f / n$ for $f \otimes \mathbb{Z} / n$ when $n \in \mathbb{Z}_{>0}$, and for $f \otimes \mathbb{Q} / \mathbb{Z}$ when $n=\infty$. Let $m, n \in \mathbb{Z}_{>0}$. The map $\operatorname{ker}(f / n) \rightarrow \operatorname{ker}(f / n m)$ induced by the map $m: A \rightarrow A$ is designated the canonical map, so that $\{\operatorname{ker}(f / n)\}_{n}$ becomes an inductive system whose limit is $\operatorname{ker}(f \otimes \mathbb{Q} / \mathbb{Z})$. The map $\operatorname{ker}(f / n m) \rightarrow \operatorname{ker}(f / n)$ induced by the identity map on $A$ is designated the canonical map, so that $\{\operatorname{ker}(f / n)\}_{n}$ becomes an inverse system.

Let $F$ be a field. A separated scheme of finite type over $F$ is called a variety over $F$. Let $X$ be a variety over $F$. For $i \in \mathbb{Z}_{\geq 0}$, we write $X_{(i)}$ and $X^{(i)}$ for the set of all points on $X$ of dimension $i$ and of codimension $i$, respectively. We write $\bar{F}$ for an algebraic closure of $F$, and we write $\bar{X}$ for the base change $X \times{ }_{\operatorname{Spec} F} \operatorname{Spec} \bar{F}$. A closed subset of $X$ is always regarded as a reduced subvariety of $X$.

\section{$\S 2$. Wiesend's ideal class group and motivic homology}

Let $F$ be a perfect field. Except in the beginning of Section 2.1 and in Section 2.3, we will assume that the characteristic of $F$ is zero. 


\subsection{Motivic homology and cohomology}

We recall some facts from [42] and [26]. Let $\mathrm{DM}_{\text {Nis }}^{\text {eff,- }}(F)$ denote the rigid triangulated tensor category of effective motivic complexes (see [42, Section 3.1], [26, Definition 14.1]). There is a functor $M$ from the category of varieties over $F$ to $\operatorname{DM}_{\text {Nis }}^{\text {eff, }}(F)$. For a variety $X$ and $n \in \mathbb{Z}_{>0} \cup\{\infty\}$, we write $M(X, \mathbb{Z} / n \mathbb{Z})=M(X) \otimes^{\mathbb{L}} \mathbb{Z} / n \mathbb{Z}$ (see Section 1.5). Let $i, j \in \mathbb{Z}$. Motivic cohomology and motivic homology of a variety $X$ are defined by

$$
\begin{aligned}
& H_{M}^{i}(X, \mathbb{Z}(j))=\operatorname{Hom}_{\mathrm{DM}_{\mathrm{Nis}}^{\mathrm{eff},-}(F)}(M(X), \mathbb{Z}(j)[i]), \\
& H_{i}^{M}(X, \mathbb{Z}(j))=\operatorname{Hom}_{\mathrm{DM}_{\mathrm{Nis}}^{\mathrm{eff},-}(F)}(\mathbb{Z}(j)[i], M(X)) .
\end{aligned}
$$

For $n \in \mathbb{Z}_{>0} \cup\{\infty\}$, their coefficient versions are defined by

$$
\begin{aligned}
& H_{M}^{i}(X, \mathbb{Z} / n \mathbb{Z}(j))=\operatorname{Hom}_{\mathrm{DM}_{\mathrm{Nis}}^{\mathrm{eff},-}(k)}(M(X), \mathbb{Z} / n \mathbb{Z}(j)[i]), \\
& H_{i}^{M}(X, \mathbb{Z} / n \mathbb{Z}(j))=\operatorname{Hom}_{\mathrm{DM}_{\mathrm{Nis}}^{\mathrm{eff},-}(k)}(\mathbb{Z}(j)[i], M(X, \mathbb{Z} / n \mathbb{Z})) .
\end{aligned}
$$

They fit into (obvious) exact sequences

$$
\begin{aligned}
0 & \rightarrow H_{M}^{i}(X, \mathbb{Z}(j)) / n \\
& \rightarrow H_{M}^{i}(X, \mathbb{Z} / n \mathbb{Z}(j)) \rightarrow H_{M}^{i+1}(X, \mathbb{Z}(j))[n] \rightarrow 0, \\
0 & \rightarrow H_{i}^{M}(X, \mathbb{Z}(j)) / n \\
& \rightarrow H_{i}^{M}(X, \mathbb{Z} / n \mathbb{Z}(j)) \rightarrow H_{i-1}^{M}(X, \mathbb{Z}(j))[n] \rightarrow 0 .
\end{aligned}
$$

Motivic cohomology $H_{M}^{i}(X, \mathbb{Z}(j))$ is contravariantly functorial in $X$. It also has covariant functionality: if $f: X \rightarrow Y$ is a proper flat equidimensional morphism of relative dimension $d$, then there is an induced map $H_{M}^{i}(X, \mathbb{Z}(j)) \rightarrow H_{M}^{i-2 d}(Y, \mathbb{Z}(j-d))$. Motivic homology $H_{i}^{M}(X, \mathbb{Z}(j))$ is covariantly functorial in $X$. It also has contravariant functionality: if $f$ : $X \rightarrow Y$ is a proper flat equidimensional morphism of relative dimension $d$, then there is an induced map $H_{i}^{M}(Y, \mathbb{Z}(j)) \rightarrow H_{i+2 d}^{M}(X, \mathbb{Z}(j+d))$.

The following fact will play an important role in the proof of Theorem 1.3: for any variety $X$, there is a decomposition

$$
H_{i}^{M}\left(X \times \mathbb{G}_{m}, \mathbb{Z}(j)\right) \cong H_{i-1}^{M}(X, \mathbb{Z}(j-1)) \oplus H_{i}^{M}(X, \mathbb{Z}(j)) .
$$

This is deduced from the Mayer-Vietoris sequence, the projective bundle formula, and $\mathbb{A}^{1}$-homotopy invariance. 
From now through the end of Section 2.2, we assume that the characteristic of $F$ is zero. If $X$ is a smooth variety, we have a canonical isomorphism

$$
H_{M}^{i}(X, \mathbb{Z}(j)) \cong \mathrm{CH}^{j}(X, 2 j-i),
$$

where the right-hand side is Bloch's higher Chow group. If $X$ is a smooth projective variety of pure dimension $d$, we also have

$$
H_{i}^{M}(X, \mathbb{Z}(j)) \cong H_{M}^{2 d-i}(X, \mathbb{Z}(d-j)) \cong \mathrm{CH}^{d-j}(X, i-2 j)
$$

In particular, if $X=\operatorname{Spec} F$, then we have, for any $r \in \mathbb{Z}_{\geq 0}$,

$$
\begin{aligned}
H_{-r}^{M}(\operatorname{Spec} F, \mathbb{Z}(-r)) & \cong H_{M}^{r}(\operatorname{Spec} F, \mathbb{Z}(r)) \\
& \cong \mathrm{CH}^{r}(\operatorname{Spec} F, r) \cong K_{r}^{M} F
\end{aligned}
$$

Let $Z$ be a closed subvariety of a smooth variety $X$. Suppose that $Z$ is smooth and of pure codimension $c$. Then we have a long exact sequence

$$
\begin{aligned}
\cdots & \rightarrow H_{i+1}^{M}(X, \mathbb{Z}(j)) \rightarrow H_{i+1-2 c}^{M}(Z, \mathbb{Z}(j-c)) \\
& \rightarrow H_{i}^{M}(X \backslash Z, \mathbb{Z}(j)) \rightarrow H_{i}^{M}(X, \mathbb{Z}(j)) \rightarrow \cdots .
\end{aligned}
$$

Lemma 2.1. Let $X$ be a smooth variety of pure dimension d, and let $i, j \in \mathbb{Z}$.

(1) Suppose that $j<0, i>j+d$, or $i>2 j$. Then we have $H_{M}^{i}(X, \mathbb{Z}(j))=0$.

(2) Suppose that $j>i$. Then we have $H_{i}^{M}(X, \mathbb{Z}(j))=0$.

The same holds for the $\mathbb{Z} / n$-coefficient version for any $n \in \mathbb{Z}_{>0} \cup\{\infty\}$.

Proof. For (1), see [26, (3.6), (19.3)]. If $X$ is projective, (2) follows from (1) and (2.1.5). The general case follows by induction on $\operatorname{dim} X$ and (2.1.7).

By Lemma 2.1 and (2.1.2), we can identify $H_{i}^{M}(X, \mathbb{Z}(i)) / n=H_{i}^{M}(X$, $\mathbb{Z} / n(i))$ for any $i \in \mathbb{Z}$ and $n \in \mathbb{Z}_{>0}$, which will be frequently used without further notice.

REMARK 2.2. If $X$ is a smooth variety of pure dimension $d$, then motivic homology $H_{i}^{M}(X, \mathbb{Z}(j))$ is isomorphic to motivic cohomology with compact support $H_{M, c}^{2 d-i}(X, \mathbb{Z}(d-j))$. However, the work of Geisser (see Remark 1.4) suggests that motivic homology would be better for a further generalization, so we opt to use homology theory. 


\subsection{Motivic complex}

We recall some results on the motivic complex. Let $X$ be a smooth variety over $F$, and let $j \in \mathbb{Z}_{\geq 0}$. There is a complex $\mathbb{Z}(j)_{X}$ of Zariski sheaves on $X$ (see $[26,(3.1)]$ ), which is concentrated in degrees $\leq j$. The hypercohomology of $\mathbb{Z}(j)_{X}$ agrees with motivic cohomology (see $[26,(14.16)]$ ):

$$
H_{\text {Zar }}^{i}\left(X, \mathbb{Z}(j)_{X}\right) \cong H_{M}^{i}(X, \mathbb{Z}(j))
$$

Thanks to the recent resolution of Bloch-Kato conjecture by Rost and Voevodsky (see [37], [43], [44]), the following important result of Suslin and Voevodsky [39] (see also Geisser and Levine [14]) holds unconditionally.

TheOREM 2.3 ([14], [39]). Let $X$ be a smooth variety over $F$, and let

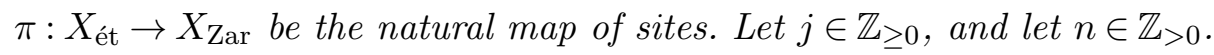

(1) There is a canonical isomorphism

$$
\pi^{*} \mathbb{Z}(j)_{X} \otimes^{\mathbb{L}} \mathbb{Z} / n \cong \mu_{n}^{\otimes j}
$$

Consequently, we have a canonical map for any $i \in \mathbb{Z}_{\geq 0}$

$$
H_{M}^{i}(X, \mathbb{Z} / n(j)) \rightarrow H_{\text {ét }}^{i}\left(X, \mu_{n}^{\otimes j}\right) .
$$

(2) The map (2.2.1) induces an isomorphism

$$
\mathbb{Z}(j)_{X} \otimes^{\mathbb{L}} \mathbb{Z} / n \rightarrow \tau_{\leq j} R \pi_{*} \mu_{n}^{\otimes j} .
$$

Consequently, the map (2.2.2) is an isomorphism if either $i \leq j$ or $j \geq$ $\operatorname{dim} X+\operatorname{cd} F$. (Here cd means the cohomological dimension.) It is an injection if $i=j+1$.

In what follows, we frequently write $H_{\text {ét }}^{i}(X, \mathbb{Z} / n(j))$ for $H_{\text {ét }}^{i}\left(X, \mu_{n}^{\otimes j}\right)$.

\subsection{Wiesend's ideal class group}

In this section, we do not assume that Char $F=0$. We will prove Theorem 1.3 after introducing a simple lemma.

Lemma 2.4. Let $r \in \mathbb{Z}_{\geq 0}$. Let $f: X \rightarrow X^{\prime}$ be a morphism of varieties over $F$. There is a unique homomorphism $f_{*}: C_{r}(X) \rightarrow C_{r}\left(X^{\prime}\right)$ characterized by the following property: for any $x \in X_{(0)}$, the diagram

$$
\begin{array}{ccc}
K_{r}^{M} F(x) & \rightarrow & C_{r}(X) \\
\downarrow^{N_{F(x) / F(f(x))}} & & \downarrow_{f_{*}} \\
K_{r}^{M} F(f(x)) & \rightarrow & C_{r}\left(X^{\prime}\right)
\end{array}
$$


commutes. (Here the upper and lower horizontal maps are the natural map to the $x$-component and $f(x)$-component, respectively.) This makes $C_{r}$ a covariant functor on the category of varieties over $F$.

Proof. Uniqueness is clear by the definition of $C_{r}(X)$. Functoriality follows from that of the norm map of Milnor $K$-groups. We prove well-definedness. Take $y \in X_{(1)}$, and put $y^{\prime}=f(y)$. We need to show that the image of $U K_{r+1}^{M} F(y)$ in $C_{r}\left(X^{\prime}\right)$ is trivial. We consider two cases separately. First, we assume that $y^{\prime} \in X_{(0)}^{\prime}$. With the notation of Section 1.2, we regard $\bar{C}(y)$ as a curve over $F\left(y^{\prime}\right)$. Weil reciprocity (see [15, Proposition 7.4.4]) shows that the composition map

$$
K_{r+1}^{M} F(y) \stackrel{\oplus \partial_{x}}{\rightarrow} \bigoplus_{x \in \bar{C}(y)_{(0)}} K_{r}^{M} F(x) \stackrel{\oplus N_{F(x) / F\left(y^{\prime}\right)}}{\rightarrow} K_{r}^{M} F\left(y^{\prime}\right)
$$

is the zero map. In view of the definition of $U K_{r+1}^{M} F(y)$, this proves our assertion in this case. Next, we assume that $y^{\prime} \in X_{(1)}^{\prime}$, so that $f$ induces a morphism $f_{y}: \bar{C}(y) \rightarrow \bar{C}\left(y^{\prime}\right)$ such that $f_{y}^{-1}\left(C_{\infty}\left(y^{\prime}\right)\right) \subset C_{\infty}(y)$. It suffices to show that the image of $U K_{r+1}^{M} F(y)$ by the norm map $K_{r+1}^{M} F(y) \rightarrow$ $K_{r+1}^{M} F\left(y^{\prime}\right)$ is contained in $U K_{r+1}^{M} F\left(y^{\prime}\right)$. This follows from the following basic fact on the tame symbol. (We omit its proof.)

LEMMA 2.5. Let $L / K$ be a finite extension of fields. Let $v$ be a discrete valuation on $K$, and let $\left\{v_{1}, \ldots, v_{f}\right\}$ be the set of all extensions of $v$ to $L$. We write $C$ and $D_{i}(1 \leq i \leq f)$ for the residue field of $v$ and $v_{i}$, respectively. Let $r \in \mathbb{Z}_{>0}$. For any $\lambda \in K_{r}^{M} L$, we have

$$
\partial_{v} N_{L / K} \lambda=\sum_{i=1}^{f} N_{D_{i} / C} \partial_{v_{i}} \lambda
$$

Proof of Theorem 1.3. The case $r=0$ is a conjunction of the comparison theorem of motivic homology with Suslin's homology (see [42, Corollary 3.2.7], [26, Proposition 14.18]) and the comparison theorem of Suslin's homology and $C_{0}(V)$ (see [34, Theorem 5.1]). We proceed by induction on $r$. 
We construct a commutative diagram

$$
\begin{array}{ccc}
\bigoplus_{y \in\left(V \times \mathbb{G}_{m}\right)_{(1)}} U K_{r+1}^{M} F(y) & \stackrel{\partial}{\rightarrow} & \bigoplus_{x \in\left(V \times \mathbb{G}_{m}\right)_{(0)}} K_{r}^{M} F(x) \\
\downarrow & \downarrow_{g} \\
\bigoplus_{w \in V_{(1)}} U K_{r+2}^{M} F(w) & \stackrel{\partial}{\rightarrow} & \bigoplus_{z \in V_{(0)}} K_{r+1}^{M} F(z)
\end{array}
$$

The two horizontal maps $\partial$ are given by the tame symbol. For $x \in(V \times$ $\left.\mathbb{G}_{m}\right)_{(0)}$ and $z \in V_{(0)}$, the $(x, z)$-component of $g$ is given as follows. It is the zero map if $z \neq p_{0}(x)$, where $p_{0}: V \times \mathbb{G}_{m} \rightarrow V$ is the projection. Suppose that $z=p_{0}(x)$, so that $F(x)$ is a finite extension of $F(z)$. The composition $x \rightarrow V \times \mathbb{G}_{m} \rightarrow \mathbb{G}_{m}$ defines an element $\xi(x) \in \mathbb{G}_{m}(F(x))=F(x)^{*}$. Now the map in question is given by

$$
K_{r}^{M} F(x) \stackrel{\text { mult. by }}{\rightarrow} \xi(x) K_{r+1}^{M} F(x) \stackrel{N_{F(x) / F(z)}}{\rightarrow} K_{r+1}^{M} F(z) .
$$

Next, let $y \in\left(V \times \mathbb{G}_{m}\right)_{(1)}$, and let $w \in V_{(1)}$. If $w \neq p_{0}(y)$, then the $(y, w)$ component of $f$ is the zero map. Suppose that $w=p_{0}(y)$, so that $F(y)$ is a finite extension of $F(w)$. The composition $y \rightarrow V \times \mathbb{G}_{m} \rightarrow \mathbb{G}_{m}$ defines an element $\xi(y) \in \mathbb{G}_{m}(F(y))=F(y)^{*}$. Now the $(y, w)$-component of $f$ is given by

$$
U K_{r+1}^{M} F(y) \stackrel{\text { mult. by } \xi(y)}{\rightarrow} U K_{r+2}^{M} F(y) \stackrel{N_{F(y) / F(w)}}{\rightarrow} U K_{r+2}^{M} F(w) .
$$

We check the commutativity. Let $y \in\left(V \times \mathbb{G}_{m}\right)_{(1)}$, and let $\lambda \in U K_{r+1}^{M} F(y)$. Put $w=p_{0}(y)$. When $w \in V_{(0)}$, by definition we have $\partial(f(\lambda))=0$, and setting $z=w$, we have

$$
\begin{aligned}
g(\partial(\lambda)) & =g\left(\sum_{x \in C(y)} \partial_{x} \lambda\right)=\sum_{x \in \tilde{C}(y)} N_{F(x) / F(z)}\left\{\partial_{x} \lambda, \xi(x)\right\} \\
& \stackrel{(1)}{=} \sum_{x \in \tilde{C}(y)} N_{F(x) / F(z)} \partial_{x}\{\lambda, \xi(y)\} \\
& \stackrel{(2)}{=}\left[\sum_{x \in \tilde{C}(y)}+\sum_{x \in C_{\infty}(y)}\right]\left(N_{F(x) / F(z)} \partial_{x}\{\lambda, \xi(y)\}\right) \\
& \stackrel{(3)}{=} 0 .
\end{aligned}
$$

Here, at (1) we used the fact that for all $x \in \tilde{C}(y)$ we have $\operatorname{ord}_{x} \xi(y)=0$ and $\partial_{x}\left\{\xi(y), \pi_{x}\right\}=\xi(x)$, where $\pi_{x} \in k(y)^{*}$ is a uniformizer at $x$. At $(2)$, we 
used the definition of $U K_{r+1}^{M} F(y)$ (i.e., $\partial_{x}(\lambda)=0$ and $\partial_{x}\left\{\lambda, \pi_{x}\right\}=0$ for all $x \in C_{\infty}(y)$ ). The equality (3) is Weil reciprocity (see [15, Proposition 7.4.4]). Now we suppose that $w \in V_{(1)}$. We take $z \in V_{(0)}$, and we write $\left\{x_{1}, \ldots, x_{n}\right\}$ for the set of all points on $\tilde{C}(y)$ above $z$. We have

$$
\begin{aligned}
z \text {-component of } \partial(f(\lambda)) & =\partial_{z} N_{F(y) / F(w)}\{\lambda, \xi(y)\} \\
& \stackrel{(1)}{=} \sum_{i=1}^{n} N_{F\left(x_{i}\right) / F(z)} \partial_{x_{i}}\{\lambda, \xi(y)\} \\
& \stackrel{(2)}{=} \sum_{i=1}^{n} N_{F\left(x_{i}\right) / F(z)}\left\{\partial_{x_{i}}(\lambda), \xi\left(x_{i}\right)\right\} \\
& =z \text {-component of } g(\partial(\lambda)) .
\end{aligned}
$$

At (1), we used Lemma 2.5. The equality (2) follows from the fact that $\operatorname{ord}_{x} \xi(y)=0$ and $\partial_{x}\left\{\xi(y), \pi_{x}\right\}=\xi(x)$ for all $x \in \tilde{C}(y)$. This proves the commutativity.

It can be seen without difficulty that both $f$ and $g$ are surjective. As a consequence, we get a surjection

$$
\alpha: C_{r}\left(V \times \mathbb{G}_{m}\right) \rightarrow C_{r+1}(V) .
$$

It follows from the definition that the composition $C_{r}(V)=C_{r}(V \times\{1\}) \rightarrow$ $C_{r}\left(V \times \mathbb{G}_{m}\right) \stackrel{\alpha}{\rightarrow} C_{r+1}(V)$ is the zero map.

Next, we consider a diagram (see $[20,(4.3)]$ )

$$
\bigoplus_{x \in\left(V \times \mathbb{G}_{m}\right)_{(0)}} K_{r}^{M} F(x) \rightarrow H_{-r}^{M}\left(V \times \mathbb{G}_{m}, \mathbb{Z}(-r)\right)
$$

$$
\begin{array}{ccc}
\downarrow^{g} & \downarrow \\
\bigoplus_{z \in V_{(0)}} K_{r+1}^{M} F(z) & \rightarrow & H_{-r-1}^{M}(V, \mathbb{Z}(-r-1))
\end{array}
$$

Here the horizontal maps are induced by the functoriality of motivic homology and (2.1.6). The right vertical map is the projection with respect to the decomposition (2.1.3). The left vertical map $g$ was defined above.

We denote by $P_{r}(V)$ the assertion that the diagram (2.3.2) is commutative. We also denote by $Q_{r}(V)$ the assertion that $\bigoplus_{x \in V_{(0)}} K_{r}^{M} F(x) \rightarrow$ $H_{-r}^{M}(V, \mathbb{Z}(-r))$ induces an isomorphism $C_{r}(V) \cong H_{-r}^{M}(V, \mathbb{Z}(-r)$ ) (which is what the theorem claims). We claim the following. 
(1) We have $Q_{0}(V)$ for all $V$.

(2) Fix $r \in \mathbb{Z}_{\geq 0}$ and a finite extension $F^{\prime} / F$. If $Q_{r}\left(\mathbb{G}_{m} \times \operatorname{Spec} F^{\prime}\right)$ holds, then $P_{r}\left(\operatorname{Spec} F^{\prime}\right)$ holds.

(3) Fix $r \in \mathbb{Z}_{\geq 0}$. If $P_{r}\left(\operatorname{Spec} F^{\prime}\right)$ holds for any finite extension $F^{\prime} / F$, then $P_{r}(V)$ holds for all $V$.

(4) Fix $r \in \mathbb{Z}_{\geq 0}$ and $V$. If $P_{r}(V), Q_{r}(V)$, and $Q_{r}\left(V \times \mathbb{G}_{m}\right)$ hold, then $Q_{r+1}(V)$ holds.

Indeed, claim (1) was already remarked at the beginning of the proof. Claim (2) holds because we have $Q_{r+1}\left(\operatorname{Spec} F^{\prime}\right)$ by (2.1.6). (By the definition of $C_{r}(V)$, if one has $Q_{r}\left(V \times \mathbb{G}_{m}\right)$ and $Q_{r+1}(V)$, then $P_{r}(V)$ becomes trivial.) Claim (3) is clear from the definition of $P_{r}(V)$. We prove claim (4). By $P_{r}(V), Q_{r}\left(V \times \mathbb{G}_{m}\right)$, and the surjectivity of $f$, we get a well-defined map (!) in the commutative diagram

$$
\begin{array}{ccc}
C_{r}\left(V \times \mathbb{G}_{m}\right) / C_{r}(V) & \stackrel{\alpha}{ } & C_{r+1}(V) \\
\downarrow & & \downarrow(!) \\
H_{-r}^{M}\left(V \times \mathbb{G}_{m}, \mathbb{Z}(-r)\right) / H_{-r}^{M}(V, \mathbb{Z}(-r)) & \cong & H_{-r-1}^{M}(V, \mathbb{Z}(-r-1))
\end{array}
$$

By $Q_{r}(V)$ and $Q_{r}\left(V \times \mathbb{G}_{m}\right)$, the left vertical map is an isomorphism. It follows that the right vertical map is also an isomorphism, which shows claim (4). Now the theorem follows by induction.

\section{§3. Étale cohomology with compact support}

In this section, $F$ is a field of characteristic zero.

3.1. The map $c_{X, n}^{i, j}$

Let $V$ be a smooth irreducible variety over $F$ of dimension $d$. Schmidt and Spieß [35, Proposition 3.1 and Remarks, p. 26] constructed a canonical homomorphism

$$
c_{V, n}^{i, j}: H_{2 d-i}^{M}(V, \mathbb{Z} / n(d-j)) \rightarrow H_{\text {ét }, c}^{i}(V, \mathbb{Z} / n(j))
$$

for any $i, j \in \mathbb{Z}_{\geq 0}$ and $n \in \mathbb{Z}_{>0}$, which is functorial in $V$. If $V$ is projective, $c_{V, n}^{i, j}$ coincides with the map (2.2.2) under the identification (2.1.5) and $H_{\text {ét }, c}^{i}(V, \mathbb{Z} / n(j))=H_{\text {ét }}^{i}(V, \mathbb{Z} / n(j))$. The maps $c_{X, n}^{i, j}$ are functorial with respect to the sequences (2.1.7) and the Gysin sequence in étale cohomology. By taking the inductive limit, we also have

$$
c_{V, \infty}^{i, j}: H_{2 d-i}^{M}(V, \mathbb{Q} / \mathbb{Z}(d-j)) \rightarrow H_{\text {ét }, c}^{i}(V, \mathbb{Q} / \mathbb{Z}(j)) .
$$


The source of the map $c_{V, n}^{2 d+r, d+r}$ can be identified with $C_{r}(V) / n$ by Theorem 1.3.

Remark 3.1. In [35, Proposition 3.1 and Remarks (b), p. 26], this homomorphism is constructed when $k$ is a finite field. In [35, Remarks (a), p. 26], it is pointed out that the same construction works over any perfect field, by using relative Poincaré duality instead of its absolute version. When $k$ is a $p$-adic field, one can also use the absolute version (based on $\left.H_{\mathrm{Gal}}^{2}(k, \mathbb{Z} / n \mathbb{Z}(1)) \cong \mathbb{Z} / n\right)$.

Proposition 3.2. Let $V$ be a smooth variety over $F$ of dimension $d$, let $i, j \in \mathbb{Z}_{\geq 0}$, and let $n \in \mathbb{Z}_{>0}$. If $i \leq j$ or $j \geq d+\operatorname{cd} F$, then $c_{V, n}^{i, j}$ is an isomorphism. If $i=j+1$, then $c_{V, n}^{i, j}$ is an injection.

Proof. By induction on $\operatorname{dim} V$ and (2.1.7), this can be reduced to the case where $V$ is projective, which is proved in Theorem 2.3 .

\subsection{Curves}

Let $X$ be a smooth projective irreducible curve over $F$, and let $U$ be an open dense subscheme of $X$. Put $Z=X \backslash U$.

LEMMA 3.3. Let $n \in \mathbb{Z}_{>0} \cup\{\infty\}$.

(1) We have $\operatorname{ker}\left(c_{U, n}^{2,1}\right)=0$. The map $\operatorname{coker}\left(c_{U, n}^{2,1}\right) \rightarrow \operatorname{coker}\left(c_{X, n}^{2,1}\right)$ is injective.

(2) We have $\operatorname{ker}\left(c_{U, n}^{3,2}\right)=0$. If $\operatorname{cd} F \leq 2$, then we have $\operatorname{coker}\left(c_{U, n}^{3,2}\right) \cong$ $\operatorname{coker}\left(c_{X, n}^{3,2}\right)$.

Proof. We consider a commutative diagram with exact rows

$$
\begin{aligned}
& H_{1}^{M}(X, \mathbb{Z} / n(0)) \quad \rightarrow \quad C_{1}(Z) / n \quad \rightarrow \quad C_{0}(U) / n \quad \rightarrow \quad C_{0}(X) / n \quad \rightarrow 0 \\
& \downarrow^{c_{X, n}^{1,1}} \quad \downarrow^{c_{Z, n}^{1,1}} \quad \downarrow^{c^{2,1}, n} \quad \downarrow^{c_{X, n}^{2,1}} \\
& H_{\text {êt }}^{1}(X, \mathbb{Z} / n(1)) \quad \rightarrow \quad H_{\text {èt }}^{1}(Z, \mathbb{Z} / n(1)) \stackrel{(*)}{\rightarrow} H_{\text {êt }, c}^{2}(U, \mathbb{Z} / n(1)) \rightarrow H_{\text {ét }}^{2}(X, \mathbb{Z} / n(1)) .
\end{aligned}
$$

The two left vertical maps are bijective and the two right vertical maps injective by Proposition 3.2, and (1) follows. The proof of (2) is similar.

\subsection{Surfaces}

Let $X$ be a smooth projective irreducible surface over $F$. Let $V \subset X$ be an open subvariety such that $\operatorname{dim}(X \backslash V)=0$. Let $U \subset V$ be an open subvariety such that $V \backslash U$ is a (not necessary connected) smooth curve. Given a smooth surface $U$, one can always find such $V$ and $X$. Let $C$ be the smooth compactification of $V \backslash U$ (i.e., the normalization of $X \backslash U$ ). 
Proposition 3.4. Let $n \in \mathbb{Z}_{>0} \cup\{\infty\}$.

(1) There is a canonical homomorphism

$$
\eta_{n}: \operatorname{coker}\left(c_{V, n}^{3,2}\right) \rightarrow \operatorname{coker}\left(c_{V \backslash U, n}^{3,2}\right)
$$

for which there are exact sequences

$$
\begin{gathered}
\operatorname{ker}\left(\eta_{n}\right) \stackrel{(*)}{\rightarrow} \operatorname{ker}\left(c_{U, n}^{4,2}\right) \rightarrow \operatorname{ker}\left(c_{X, n}^{4,2}\right), \\
\operatorname{coker}\left(\eta_{n}\right) \stackrel{(* *)}{\rightarrow} \operatorname{coker}\left(c_{U, n}^{4,2}\right) \rightarrow \operatorname{coker}\left(c_{V, n}^{4,2}\right) .
\end{gathered}
$$

If $H_{\text {ét }, c}^{3}(V, \mathbb{Z} / n(2)) \rightarrow H_{\text {ét }, c}^{3}(V \backslash U, \mathbb{Z} / n(2))$ is injective, then $(*)$ is injective, too. If $c_{X, n}^{4,2}$ is injective, then $(* *)$ is injective, too. If $\operatorname{cd} F \leq 2$, then we have $\operatorname{coker}\left(c_{V \backslash U, n}^{3,2}\right) \cong \operatorname{coker}\left(c_{C, n}^{3,2}\right), \operatorname{coker}\left(c_{V, n}^{3,2}\right) \cong \operatorname{coker}\left(c_{X, n}^{3,2}\right)$, and $\operatorname{coker}\left(c_{V, n}^{4,2}\right) \cong \operatorname{coker}\left(c_{X, n}^{4,2}\right)$. (That is, one can replace $V \backslash U$ and $V$ by $C$ and $X$ in the above sequences.)

(2) Suppose that $F$ is a finite extension of $\mathbb{Q}_{p}$. Then there is an exact sequence

$$
\operatorname{coker}\left(c_{X, n}^{4,3}\right) \rightarrow \operatorname{ker}\left(c_{U, n}^{5,3}\right) \rightarrow \operatorname{ker}\left(c_{X, n}^{5,3}\right),
$$

and we have $\operatorname{coker}\left(c_{U, n}^{5,3}\right) \cong \operatorname{coker}\left(c_{X, n}^{5,3}\right)$.

Before we give a proof of this proposition, we record a well-known lemma which describes the kernel and cokernel of $c_{X, n}^{i, j}$. We set $\mathcal{H}^{i}(\mathbb{Z} / n(j)):=$ $R^{i} \pi_{*} \mu_{n}^{\otimes j}$ for $n \in \mathbb{Z}_{>0} \cup\{\infty\}$ and $i, j \in \mathbb{Z}$. (See Theorem 2.3 for the definition of $\pi$.)

\section{LEMMA 3.5.}

(1) Let $n \in \mathbb{Z}_{>0} \cup\{\infty\}$. There is an exact sequence

$$
\begin{aligned}
0 & \rightarrow H_{1}^{M}(X, \mathbb{Z} / n(0)) \stackrel{c_{X, n}^{3,2}}{\rightarrow} H_{\text {ét }}^{3}(X, \mathbb{Z} / n \mathbb{Z}(2)) \\
& \rightarrow H_{\text {Zar }}^{0}\left(X, \mathcal{H}^{3}(\mathbb{Z} / n(2))\right) \rightarrow C_{0}(X) / n \stackrel{c_{X, n}^{4,2}}{\rightarrow} H_{\text {ét }}^{4}(X, \mathbb{Z} / n(2)) .
\end{aligned}
$$

(2) Let $n \in \mathbb{Z}_{>0} \cup\{\infty\}$. There is an exact sequence

$$
\begin{aligned}
0 & \rightarrow H_{0}^{M}(X, \mathbb{Z} / n(-1)) \stackrel{c_{X, n}^{4,3}}{\rightarrow} H_{\text {ét }, c}^{4}(X, \mathbb{Z} / n(3)) \rightarrow H_{\text {Zar }}^{0}\left(X, \mathcal{H}^{4}(\mathbb{Z} / n(3))\right) \\
& \rightarrow C_{1}(X) / n \stackrel{c_{X, n}^{5,3}}{\rightarrow} H_{\text {ét }, c}^{5}(X, \mathbb{Z} / n(3)) \rightarrow H_{\text {Zar }}^{1}\left(X, \mathcal{H}^{4}(\mathbb{Z} / n(3))\right) \rightarrow 0 .
\end{aligned}
$$


(3) Let $n \in \mathbb{Z}_{>0}$. The canonical map $H_{\mathrm{Zar}}^{0}\left(X, \mathcal{H}^{i}(\mathbb{Z} / n(i-1))\right) \rightarrow H_{\text {Zar }}^{0}(X$, $\left.\mathcal{H}^{i}(\mathbb{Q} / \mathbb{Z}(i-1))\right)[n]$ is bijective for $i=3,4$.

Proof. Since $X$ is projective, (1) and (2) follow from (2.1.5) and the Bloch-Ogus theory; (3) is a consequence of the Bloch-Kato conjecture.

Proof of Proposition 3.4(1). We have a commutative diagram with exact rows

$$
\begin{aligned}
& H_{1}^{M}(V, \mathbb{Z} / n \mathbb{Z}(0)) \quad \rightarrow \quad C_{1}(V \backslash U) / n \quad \rightarrow \quad C_{0}(U) / n \quad \rightarrow \quad C_{0}(V) / n \rightarrow 0 \\
& \downarrow^{c_{V, n}^{3,2}} \quad \downarrow^{c^{3,2},{ }^{2}} \quad \downarrow^{c_{U, n}^{4,2}} \quad \downarrow^{c_{V, n}^{4,2}} \\
& H_{\text {ét }, c}^{3}(V, \mathbb{Z} / n \mathbb{Z}(2)) \rightarrow H_{\text {ét }, c}^{3}(V \backslash U, \mathbb{Z} / n \mathbb{Z}(2)) \rightarrow H_{\text {ét }, c}^{4}(U, \mathbb{Z} / n \mathbb{Z}(2)) \rightarrow H_{\text {ét }, c}^{4}(V, \mathbb{Z} / n \mathbb{Z}(2))
\end{aligned}
$$

Proposition 3.2 shows the injectivity of $c_{V, n}^{3,2}$ and $c_{V \backslash U, n}^{3,2}$. If $\operatorname{cd} F \leq 2$, then we have $\operatorname{coker}\left(c_{V \backslash U, n}^{3,2}\right) \cong \operatorname{coker}\left(c_{C, n}^{3,2}\right)$ by Lemma 3.3. Using the following lemma, a diagram chase completes the proof.

Lemma 3.6. The map $\operatorname{ker}\left(c_{V, n}^{4,2}\right) \rightarrow \operatorname{ker}\left(c_{X, n}^{4,2}\right)$ is injective. If $\operatorname{cd} F \leq 2$, then $\operatorname{coker}\left(c_{V, n}^{i, 2}\right) \rightarrow \operatorname{coker}\left(c_{X, n}^{i, 2}\right)$ is bijective for $i=3,4$.

Proof. This follows from Lemma 2.1, Proposition 3.2, and (2.1.7).

Proof of Proposition 3.4(2). We have a commutative diagram with exact rows

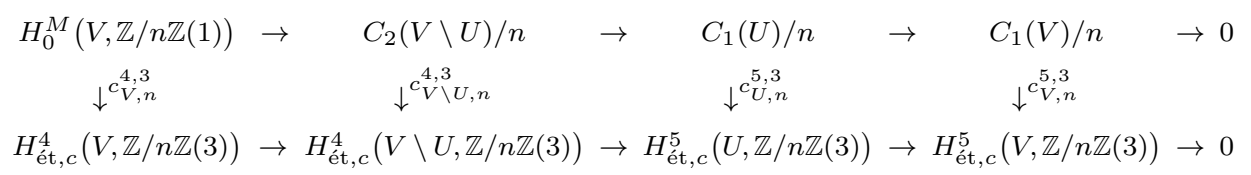

Proposition 3.2 shows the injectivity of the two left vertical maps. In Proposition 4.3 below, we will show that $c_{V \backslash U, n}^{4,3}$ is bijective. Using the following lemma, a diagram chase completes the proof.

Lemma 3.7. The map $\operatorname{ker}\left(c_{V, n}^{5,3}\right) \rightarrow \operatorname{ker}\left(c_{X, n}^{5,3}\right)$ is injective. If $\operatorname{cd} F \leq 2$, then $\operatorname{coker}\left(c_{V, n}^{i, 3}\right) \rightarrow \operatorname{coker}\left(c_{X, n}^{i, 3}\right)$ is bijective for $i=3,4$.

Proof. This follows from Lemma 2.1, Proposition 3.2, and (2.1.7).

\section{$\S 4$. Varieties over a $p$-adic field}

In this section, $k$ is a finite extension of $\mathbb{Q}_{p}$. Let $X$ be a smooth geometrically irreducible variety over $k$ of dimension $d$. 


\subsection{The Brauer-Manin pairing}

By Poincaré duality, we have a canonical isomorphism

$$
H_{\text {ét }, c}^{2 d}(X, \hat{\mathbb{Z}}(d)) \cong H_{\text {ét }}^{2}(X, \mathbb{Q} / \mathbb{Z}(1))^{*} .
$$

Since $\operatorname{Br}(X)$ is a torsion group, the Kummer sequence implies an exact sequence

$$
0 \rightarrow \operatorname{Pic}(X) \otimes \mathbb{Q} / \mathbb{Z} \rightarrow H_{\text {èt }}^{2}(X, \mathbb{Q} / \mathbb{Z}(1)) \rightarrow \operatorname{Br}(X) \rightarrow 0 ;
$$

hence, we have an injective homomorphism $\operatorname{Br}(X)^{*} \rightarrow H_{\text {ét, }}^{2 d}(X, \hat{\mathbb{Z}}(d))$. We consider a diagram

$$
\begin{array}{ccc}
Z_{0}(X) & \stackrel{\tilde{\psi}_{X}}{\rightarrow} & \operatorname{Br}(X)^{*} \\
\downarrow^{\text {surj. }} & & \downarrow_{\text {inj. }} \\
C_{0}(X) & \rightarrow & H_{\text {ét, }, c}^{2 d}(X, \hat{\mathbb{Z}}(d))
\end{array}
$$

where the lower horizontal map is the composition of

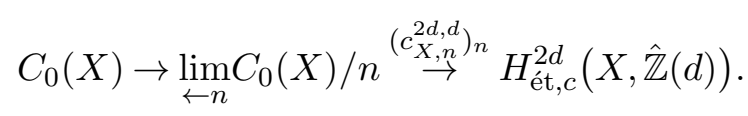

This diagram is seen to be commutative by reducing to the case $\operatorname{dim} X=0$. This shows the first part of Proposition 1.5 (the well-definedness of $\psi_{X}$ ). The same argument shows the following simple lemma.

Lemma 4.1. For any $n \in \mathbb{Z}_{>0} \cup\{\infty\}$, we have an isomorphism $\operatorname{ker}\left(\psi_{X} /\right.$ $n) \cong \operatorname{ker}\left(c_{X, n}^{2 d, d}\right)$ and an exact sequence

$$
0 \rightarrow \operatorname{coker}\left(\psi_{X} / n\right) \rightarrow \operatorname{coker}\left(c_{X, n}^{2 d, d}\right) \rightarrow(\operatorname{Pic}(X) / n)^{*} \rightarrow 0 .
$$

\subsection{Class field theory}

By Poincaré duality, we have a canonical isomorphism

$$
H_{\text {ét }, c}^{2 d+1}(X, \hat{\mathbb{Z}}(d+1)) \cong H_{\text {ét }}^{1}(X, \mathbb{Q} / \mathbb{Z})^{*} \cong \pi_{1}^{\mathrm{ab}}(X) .
$$

We consider a diagram

$$
\begin{array}{ccc}
Z_{0}^{1}(X) \stackrel{\tilde{\rho}_{X}}{\rightarrow} & \pi_{1}^{\mathrm{ab}}(X) \\
\downarrow^{\text {surj. }} & \downarrow \cong \\
C_{1}(X) & \rightarrow & H_{\text {ét, }, c}^{2 d+1}(X, \hat{\mathbb{Z}}(d+1))
\end{array}
$$


where the lower horizontal map is the composition of

$$
C_{1}(X) \rightarrow \lim _{\leftarrow n} C_{1}(X) / n \stackrel{\left(c_{X, n}^{2 d+1, d+1}\right)_{n}}{\rightarrow} H_{\text {ét,c }}^{2 d+1}(X, \hat{\mathbb{Z}}(d+1)) .
$$

This diagram is seen to be commutative by reducing to the case $\operatorname{dim} X=0$. This shows the second part of Proposition 1.5 (the well-definedness of $\rho_{X}$ ). The same argument shows the following simple lemma.

Lemma 4.2. For any $n \in \mathbb{Z}_{>0} \cup\{\infty\}$, we have isomorphisms $\operatorname{ker}\left(\rho_{X} / n\right) \cong$ $\operatorname{ker}\left(c_{X, n}^{2 d+1, d+1}\right)$ and $\operatorname{coker}\left(\rho_{X} / n\right) \cong \operatorname{coker}\left(c_{X, n}^{2 d+1, d+1}\right)$.

\subsection{Higher degree}

We consider the groups $C_{r}(X)$ when $r \geq 2$. Proposition 4.3 below shows that $C_{r}(X)$ is uniquely divisible if $r \geq 3$ and that $C_{2}(X) \rightarrow C_{2}(\operatorname{Spec} k)=$ $K_{2}(k)$ is a surjective map with uniquely divisible kernel. Note that $K_{2}(k)$ is the direct sum of a uniquely divisible group and a finite group isomorphic to $\mu(k)$ (see $[27])$.

Proposition 4.3. Let $X$ be a smooth geometrically irreducible variety over $k$. Suppose that there exists a smooth projective variety $Y$ that contains $X$ as an open dense subvariety. Let $i, j \in \mathbb{Z}$, and suppose that $j \leq-2$.

(1) If $i<-2$, then $H_{i}^{M}(X, \mathbb{Z}(j))$ is uniquely divisible.

(2) The map $H_{-2}^{M}(X, \mathbb{Z}(j)) \rightarrow H_{-2}^{M}(\operatorname{Spec} k, \mathbb{Z}(j))$ induced by the structure morphism is surjective with uniquely divisible kernel. Consequently,

$$
c_{X, n}^{2 d+2, d+2}: C_{2}(X) / n \rightarrow H_{\text {ét }, c}^{2 d+2}(X, \mathbb{Z} / n(d+2))
$$

is bijective for any $n \in \mathbb{Z}_{>0}$.

Proof. Since $j \leq-2$, Proposition 3.2 shows that

$$
c_{X, n}^{2 d-i, d-j}: H_{i}^{M}(X, \mathbb{Z} / n(j)) \rightarrow H_{\text {ét, }, c}^{2 d-i}(X, \mathbb{Z} / n(d-j))
$$

is an isomorphism for any $n \in \mathbb{Z}_{>0} \cup\{\infty\}$. If $i<-2$, then we have $H_{\text {ét }, c}^{2 d-i}(X$, $\mathbb{Z} / n \mathbb{Z}(d-j))=0$ since $\operatorname{cd} k=2$. We also have $H_{\text {ét }, c}^{2 d+2}(X, \mathbb{Q} / \mathbb{Z}(d-j))=0$, because this group is dual to $H_{\text {Gal }}^{0}(k, \hat{\mathbb{Z}}(j+1))^{*}=0$ under Poincaré duality. Now (1) follows from (2.1.2).

Proposition 4.3(2) follows from the four claims (a)-(d) below. In what follows, we write $H_{*}^{M}(k,-)$ for $H_{*}^{M}(\operatorname{Spec} k,-)$. Put $H_{-2}^{M}(X, \mathbb{Z}(j))_{0}:=$ $\operatorname{ker}\left[H_{-2}^{M}(X, \mathbb{Z}(j)) \rightarrow H_{-2}^{M}(k, \mathbb{Z}(j))\right]$. Then we have the following: 
(a) $H_{-2}^{M}(X, \mathbb{Z}(j)) \rightarrow H_{-2}^{M}(k, \mathbb{Z}(j))$ is surjective;

(b) $H_{-2}^{M}(X, \mathbb{Z}(j)) / n \rightarrow H_{-2}^{M}(k, \mathbb{Z}(j)) / n$ is bijective for any $n \in \mathbb{Z}_{>0}$;

(c) $H_{-2}^{M}(X, \mathbb{Z}(j))[n] \rightarrow H_{-2}^{M}(k, \mathbb{Z}(j))[n]$ is surjective for any $n \in \mathbb{Z}_{>0}$;

(d) $H_{-2}^{M}(X, \mathbb{Z}(j))_{0}$ is torsion-free.

Indeed, by (a) we have an exact sequence for any $n \in \mathbb{Z}_{>0}$,

$$
\begin{aligned}
0 & \rightarrow H_{-2}^{M}(X, \mathbb{Z}(j))_{0}[n] \rightarrow H_{-2}^{M}(X, \mathbb{Z}(j))[n] \rightarrow H_{-2}^{M}(k, \mathbb{Z}(j))[n] \\
& \rightarrow H_{-2}^{M}(X, \mathbb{Z}(j))_{0} / n \rightarrow H_{-2}^{M}(X, \mathbb{Z}(j)) / n \rightarrow H_{-2}^{M}(k, \mathbb{Z}(j)) / n \rightarrow 0,
\end{aligned}
$$

which shows (2) in view of (b)-(d).

We prove claims $(\mathrm{a})-(\mathrm{d})$. Since we have shown that $H_{-3}^{M}(X, \mathbb{Z}(j))_{\text {Tor }}=0$, (2.1.2) and (4.3.1) yield a commutative diagram

$$
\begin{array}{ccc}
H_{-2}^{M}(X, \mathbb{Z}(j)) / n & \cong & H_{\text {ét }, c}^{2 d+2}(X, \mathbb{Z} / n \mathbb{Z}(d-j)) \\
\downarrow & \downarrow \cong \\
H_{-2}^{M}(k, \mathbb{Z}(j)) / n & \cong & H_{\text {Gal }}^{2}(k, \mathbb{Z} / n \mathbb{Z}(-j))
\end{array}
$$

which shows (b). Taking a closed point $x \in X$, the cokernel of $H_{-2}^{M}(X$, $\mathbb{Z}(j)) \rightarrow H_{-2}^{M}(k, \mathbb{Z}(j))$ is seen to be annihilated by $[k(x): k]$. Using (b) with $n=[k(x): k]$, we get (a). By (2.1.2) we have a commutative diagram with exact rows

$$
\begin{array}{ccc}
H_{\text {ét }, c}^{2 d+1}(X, \mathbb{Z} / n \mathbb{Z}(d-j)) & \rightarrow & H_{-2}^{M}(X, \mathbb{Z}(j))[n] \rightarrow 0 \\
(*) \downarrow & \downarrow \\
H_{\text {Gal }}^{1}(k, \mathbb{Z} / n \mathbb{Z}(-j)) & \rightarrow & H_{-2}^{M}(k, \mathbb{Z}(j))[n] \rightarrow 0
\end{array}
$$

The map $(*)$ is induced by the structure morphism $\alpha: X \rightarrow$ Spec $k$. Thus, it is dual to $\alpha^{*}: H_{\text {Gal }}^{1}(k, \mathbb{Z} / n \mathbb{Z}(1+j)) \rightarrow H_{\text {ét }}^{1}(X, \mathbb{Z} / n \mathbb{Z}(1+j))$, which is injective by Leray's spectral sequence. This shows that $(*)$ is surjective, and (c) follows. We consider a commutative diagram with exact rows and columns

$$
\begin{aligned}
& 0 \\
& \downarrow \\
& H_{\text {Gal }}^{2}\left(k, H_{\text {ét }, c}^{2 d-1}(\bar{X}, \mathbb{Q} / \mathbb{Z}(d-j))\right) \quad H_{-2}^{M}(X, \mathbb{Z}(j))_{0, \text { Tor }} \\
& \downarrow \\
& 0 \rightarrow H_{-1}^{M}(X, \mathbb{Z}(j)) \otimes \mathbb{Q} / \mathbb{Z} \rightarrow \quad H_{\text {ét }, c}^{2 d+1}(X, \mathbb{Q} / \mathbb{Z}(d-j)) \quad \rightarrow \quad H_{-2}^{M}(X, \mathbb{Z}(j))_{\text {Tor }} \rightarrow 0 \\
& 0 \rightarrow H_{-1}^{M}(k, \mathbb{Z}(j)) \otimes \mathbb{Q} / \mathbb{Z} \rightarrow \quad H_{\text {Gal }}^{1}(k, \mathbb{Q} / \mathbb{Z}(d-j)) \quad \rightarrow \quad H_{-2}^{M}(k, \mathbb{Z}(j))_{\text {Tor }} \quad \rightarrow 0
\end{aligned}
$$


Note that the lower left vertical map is surjective since the cokernel of $H_{-1}^{M}(X, \mathbb{Z}(j)) \rightarrow H_{-1}^{M}(k, \mathbb{Z}(j))$ is torsion (annihilated by $[k(x): k]$ for any closed point $x \in X)$. Now the following lemma completes the proof of $(\mathrm{d})$.

LEMMA 4.4. Let $X$ be a variety over $k$ satisfying the same assumption as in Proposition 4.3. If $j \leq-2$, then $H_{\text {Gal }}^{2}\left(k, H_{\text {ét }, c}^{2 d-1}(\bar{X}, \mathbb{Q} / \mathbb{Z}(d-j))\right)=0$.

Proof. By Poincaré duality, it suffices to show that $H_{\mathrm{Gal}}^{0}\left(k, H_{\text {ét }}^{1}\left(\bar{X}, \mathbb{Z}_{l}(j+\right.\right.$ $1)))=0$ for all prime numbers $l$. There is an exact sequence

$$
0 \rightarrow H_{\text {ét }}^{1}\left(\bar{Y}, \mathbb{Z}_{l}(j+1)\right) \rightarrow H_{\text {ét }}^{1}\left(\bar{X}, \mathbb{Z}_{l}(j+1)\right) \rightarrow H_{\text {ét }, \bar{Y} \backslash X}^{2}\left(\bar{Y}, \mathbb{Z}_{l}(j+1)\right) .
$$

By excision and purity, we have $H_{\text {Gal }}^{0}\left(k, H_{\text {et }, \bar{Y} \backslash X}^{2}\left(\bar{Y}, \mathbb{Z}_{l}(j+1)\right)\right)=0$. Thus, we are reduced to showing that $H_{\text {Gal }}^{0}\left(k, H_{\text {ét }}^{1}\left(\bar{Y}, \mathbb{Z}_{l}(j+1)\right)\right)=0$, but this is a result of Jannsen [17, Theorems 4.2 and 5.3], for the cases $l \neq p$ and $l=p$, respectively.

\subsection{Auxiliary lemmas}

For future use, we record a few simple lemmas. The following lemma is often used concurrently with the fact that the groups $\pi_{1}^{\mathrm{ab}}(X)$ and $\operatorname{Br}(X)^{*}$ have no nontrivial divisible elements for any smooth variety $X$ (see Section 1.5).

Lemma 4.5. Let $f: A \rightarrow B$ be a homomorphism of abelian groups.

(1) Suppose that $B_{\mathrm{div}}=0$. Then there is an exact sequence

$$
0 \rightarrow A_{\text {div }} \rightarrow \operatorname{ker}(f) \rightarrow \lim _{\leftarrow} \operatorname{ker}(f / n)
$$

(2) Suppose that there is an $N \in \mathbb{Z}_{>0}$ such that the canonical map $\operatorname{ker}(f / n N) \rightarrow \operatorname{ker}(f \otimes \mathbb{Q} / \mathbb{Z})$ is bijective for all $n \in \mathbb{Z}_{>0}$. Then we have $\lim _{\leftarrow} \operatorname{ker}(f / n)=0$. (Hence, $\operatorname{ker}(f)=A_{\text {div }}$ if $B_{\text {div }}=0$.)

Proof. Claim (1) is a direct consequence of the definition. We prove (2). By assumption, the canonical map $\operatorname{ker}(f / m N) \rightarrow \operatorname{ker}(f / m n N)$ is bijective for all $n, m \in \mathbb{Z}_{>0}$, which implies that the canonical map $\operatorname{ker}(f / m n N) \rightarrow$ $\operatorname{ker}(f / n N)$ is the zero map. Thus, we have $\lim _{\leftarrow} \operatorname{ker}(f / n)=0$.

Lemma 4.6. Let $X$ be a smooth irreducible variety over $k$ of dimension $d$, and let $i, j \in \mathbb{Z}$. If $i+2 \geq j+d$ or $j \geq d+2$, then we have $H_{i}^{M}(X, \mathbb{Z}(j))_{\operatorname{div}}=$ $H_{i}^{M}(X, \mathbb{Z}(j))_{\text {Div }}$. In particular, we have $C_{r}(X)_{\mathrm{div}}=C_{r}(X)_{\operatorname{Div}}$ for any $r \in$ $\mathbb{Z}_{\geq 0}$ when $d \leq 2$. If $d=1$, then $C_{0}(X)_{\text {Div }}=0$. 
Proof. It suffices to show that $H_{i}^{M}(X, \mathbb{Z}(j))[n]$ is finite for any $n \in \mathbb{Z}_{>0}$. Proposition 3.2 and $(2.1 .2)$ reduce this to the finiteness of $H_{\text {ét, } c}^{2 d-i-1}(X$, $\mathbb{Z} / n(d-j))$, which is well known. For the last statement, we recall that $C_{0}(X)$ is isomorphic to the relative Picard group (see Remark 1.2), which has no nontrivial divisible subgroup by a theorem of Mattuck [25].

\section{$\S 5$. Curves over a local field}

In this section, $k$ is a finite extension of $\mathbb{Q}_{p}$. Let $X$ be a smooth projective irreducible curve over $k$, and let $U$ be an open dense subscheme of $X$. Put $Z=X \backslash U$.

\subsection{The Brauer-Manin pairing}

The following theorem was proved by Scheiderer and van Hamel. When $U=X$, this theorem is due to Lichtenbaum [22].

THEOREM 5.1 ([33, (3.5)]). The homomorphism $\psi_{U}: C_{0}(U) \rightarrow \operatorname{Br}(U)^{*}$ is an injection with dense image. The induced map $\psi_{U} / n: C_{0}(U) / n \rightarrow$ $\operatorname{Br}(U)^{*} / n$ is an isomorphism for all $n \in \mathbb{Z}_{>0}$.

Proof. We deduce this theorem assuming Lichtenbaum's result. We may assume that $X$ is geometrically irreducible over $k$. Let $n \in \mathbb{Z}_{>0}$. Lemmas 3.3 and 4.1 show the injectivity of $\psi_{U} / n$. By Lemma 4.1, we have a commutative diagram

$$
0 \rightarrow \operatorname{coker}\left(\psi_{U} / n\right) \rightarrow \operatorname{coker}\left(c_{U, n}^{2,1}\right) \rightarrow(\operatorname{Pic}(U) / n)^{*} \rightarrow 0
$$

$$
\begin{array}{cc}
\downarrow^{(*)} & \downarrow \\
\operatorname{coker}\left(c_{X, n}^{2,1}\right) & \cong(\operatorname{Pic}(X) / n)^{*}
\end{array}
$$

The bottom horizontal map is bijective because $\operatorname{coker}\left(\psi_{X} / n\right)=0$ by Lichtenbaum's result. By Lemma 3.3, $(*)$ is injective; thus, $\operatorname{coker}\left(\psi_{U} / n\right)=0$. The rest of the assertion follows from Lemmas 4.5 and 4.6.

\subsection{Class field theory}

We recall a result for projective curves due to Bloch and Saito.

TheOREM 5.2 ([1], [29]).

(1) The kernel of the homomorphism $\rho_{X}: C_{1}(X)\left(=S K_{1}(X)\right) \rightarrow \pi_{1}^{\mathrm{ab}}(X)$ coincides with $C_{1}(X)_{\text {Div }}$. Moreover, $\rho_{X} / n$ is injective for all $n \in \mathbb{Z}_{>0}$. 
(2) The group $D(X):=\pi_{1}^{\mathrm{ab}}(X) / \overline{\operatorname{Im}\left(\rho_{X}\right)}$ is isomorphic to $\hat{\mathbb{Z}}^{\oplus r(X)}$ for some $r=r(X) \in \mathbb{Z}_{\geq 0}$. We have $\operatorname{coker}\left(\rho_{X} / n\right) \cong D(X) / n$ for any $n \in \mathbb{Z}_{>0}$.

REMARK 5.3. If $X$ has potentially good reduction, then $r(X)=0$. In general, we have an inequality $r(X) \leq g(X)$, where $g(X)$ is the genus of $X$ (see $[29,(6.2)]$ ). In particular, we have $D(X)=0$ when $g(X)=0$.

The following generalization follows from Lemmas 3.3, 4.2, 4.5, and 4.6.

THEOREM 5.4 .

(1) The kernel of the homomorphism $\rho_{U}: C_{1}(U) \rightarrow \pi_{1}^{\mathrm{ab}}(U)$ coincides with $C_{1}(U)_{\text {Div }}$. Moreover, $\rho_{U} / n$ is injective for all $n \in \mathbb{Z}_{>0}$.

(2) Set $D(U):=\pi_{1}^{\mathrm{ab}}(U) / \overline{\operatorname{Im}\left(\rho_{U}\right)}$. The canonical maps $D(U) \rightarrow D(X)$ and $\operatorname{coker}\left(\rho_{U} / n\right) \rightarrow \operatorname{coker}\left(\rho_{X} / n\right)(\cong D(X) / n)$ are bijective for any $n \in \mathbb{Z}_{>0}$.

REMARK 5.5. Following Hiranouchi [16], we define

$$
C_{1}^{w}(U):=\operatorname{coker}\left[K_{2}(k(X)) \rightarrow\left(\bigoplus_{x \in U_{(0)}} k(x)^{*}\right) \oplus\left(\bigoplus_{z \in Z} K_{2}\left(k(X)_{z}\right)\right)\right],
$$

where $k(X)_{z}$ is the completion of $k(X)$ at $z$. Hiranouchi constructed the reciprocity map $\rho_{U}^{\prime}: C_{1}^{w}(U) \rightarrow \pi_{1}^{\text {ab }}(U)$ and showed that the kernel of $\rho_{U}^{\prime}$ coincides with $C_{1}^{w}(U)_{\text {Div }}$ and that $\pi_{1}^{\mathrm{ab}}(U) / \overline{\operatorname{Im}\left(\rho_{U}^{\prime}\right)} \cong D(X)$. It is easy to see the following.

- The natural projection $\pi: C_{1}^{w}(U) \rightarrow C_{1}(U)$ fits into an exact sequence

$$
\bigoplus_{z \in Z} U K_{2} k(X)_{z} \rightarrow C_{1}^{w}(U) \stackrel{\pi}{\rightarrow} C_{1}(U) \rightarrow 0
$$

Here we put $U K_{2} k(X)_{z}=\operatorname{ker}\left[K_{2} k(X)_{z} \rightarrow k(z)^{*} \oplus K_{2} k(z)\right]$, where the map is defined by $a \mapsto\left(\partial_{z}(a), \partial_{z}\left(\left\{\pi_{z}\right\} \cup a\right)\right.$ for some uniformizer $\pi_{z} \in k(X)_{z}$ at $z$ (and $\partial_{z}$ is the tame symbol). Note that $U K_{2} k(X)_{z}$ is uniquely divisible.

- We have $\rho_{U}^{\prime}=\rho_{U} \circ \pi$.

Hence, Hiranouchi's result (see [16]) can be recovered by Theorem 5.4.

\section{$\S 6$. Surfaces over a local field}

We keep assuming that $k$ is a finite extension of $\mathbb{Q}_{p}$. Let $X$ be a smooth projective geometrically connected surface over $k$. Let $U \subset V \subset X$ be open dense subsets such that $\operatorname{dim}(X \backslash V)=0$ and such that $V \backslash U$ is a smooth curve. Let $C$ be the normalization of $X \backslash U$, and set $D(C)=\bigoplus_{i} D\left(C_{i}\right)$, where $C_{i}$ are the irreducible components of $C$, and $D\left(C_{i}\right)$ is the group defined in Theorem 5.2. 


\section{1. $\mathbb{P}^{2}$ minus curves}

As the first example, we show the following.

Proposition 6.1. Suppose that $X \cong \mathbb{P}^{2}$. Let $d$ be the greatest common divisor of the degrees (as a subvariety of $\mathbb{P}^{2}$ ) of the irreducible components of $X \backslash U$. Then, there is an exact sequence

$$
0 \rightarrow C_{0}(U) / n d \stackrel{\psi_{U} / n d}{\rightarrow} \operatorname{Br}(U)^{*} / n d \rightarrow D(C) / n d \rightarrow 0
$$

for any $n \in \mathbb{Z}_{>0}$. We have $\operatorname{ker}\left(\psi_{U}\right)=C_{0}(U)_{\text {Div }}$ and $\operatorname{Br}(U)^{*} / \overline{\operatorname{Im}\left(\psi_{U}\right)} \cong D(C)$.

For the map $\rho_{U}: C_{1}(U) \rightarrow \pi_{1}^{\mathrm{ab}}(U)$, see Theorem 6.5 below.

Proof. Let $n \in \mathbb{Z}_{>0}$. Note that $\psi_{X} / n: C_{0}(X) / n \rightarrow \operatorname{Br}(X)^{*} / n$ is bijective and that $H_{\mathrm{Zar}}^{0}\left(X, \mathcal{H}^{i}(\mathbb{Q} / \mathbb{Z}(i-1))\right)=0$ for $i=3,4$. By Lemmas 3.5 and 4.1 and Proposition 3.4, we have $\operatorname{ker}\left(\psi_{U} / n\right)=0$. By Lemmas 4.5 and 4.6, we get $\operatorname{ker}\left(\psi_{U}\right)=C_{0}(U)_{\text {Div }}$.

Note that $\mathbb{Z}=\operatorname{Pic}(X) \rightarrow \operatorname{Pic}(U)$ is a surjection with kernel $d \mathbb{Z}$. By Lemma 4.1, we get a commutative diagram with exact rows

$$
\begin{aligned}
& 0 \rightarrow \operatorname{coker}\left(\psi_{U} / n d\right) \rightarrow \operatorname{coker}\left(c_{U, n d}^{4,2}\right) \rightarrow \mathbb{Z} / d \mathbb{Z} \rightarrow 0 \\
& \downarrow \quad \downarrow^{\text {inj. }} \\
& 0=\operatorname{coker}\left(\psi_{X} / n d\right) \rightarrow \operatorname{coker}\left(c_{X, n d}^{4,2}\right) \cong \mathbb{Z} / n d
\end{aligned}
$$

By Proposition 3.4 and Lemma 3.5, we get an exact sequence

$$
0 \rightarrow \operatorname{coker}\left(c_{C, n d}^{3,2}\right) \rightarrow \operatorname{coker}\left(c_{U, n d}^{4,2}\right) \rightarrow \operatorname{coker}\left(c_{X, n d}^{4,2}\right) .
$$

It follows that $\operatorname{coker}\left(\psi_{U} / n d\right) \cong \operatorname{coker}\left(c_{C, n d}^{3,2}\right)$, which is isomorphic to $D(C) / n d$ by Lemma 4.2 and Theorem 5.2. This completes the proof.

\subsection{Rational surface}

Suppose that $\bar{X}:=X \times_{\operatorname{Spec} k}$ Spec $\bar{k}$ is a rational surface over $\bar{k}$ (i.e., $\bar{X}$ is birational to $\left.\mathbb{P}_{\bar{k}}^{2}\right)$ and that $X(k) \neq \phi$. In this situation, the Chow group $C_{0}(X)=\mathrm{CH}_{0}(X)$ and related maps such as $\psi_{X}$ and $c_{X, n}^{4,2}$ have been studied by several authors (see, e.g., [2]-[5], [8], [24]). We will briefly recall a few results among them.

We set $A_{0}(X):=\operatorname{ker}\left[C_{0}(X)=\mathrm{CH}_{0}(X) \stackrel{\operatorname{deg}}{\rightarrow} \mathbb{Z}\right]$. Let $S=\operatorname{Hom}\left(N S(\bar{X}), \bar{k}^{*}\right)$ be the Néron-Severi torus of $X$. Recall that we have the Bloch map (see $[2],[8])$

$$
\phi_{X}: A_{0}(X) \rightarrow H_{\mathrm{Gal}}^{1}(k, S)
$$


It is known by [3] that $\phi_{X}$ is injective. Note that $H_{\text {Gal }}^{1}(k, S)$ is finite (hence, so is $\left.A_{0}(X)\right)$. We will also need the following result.

Theorem 6.2 (Saito [30]; see also [4]). There is an $N=N_{X} \in \mathbb{Z}_{>0}$ such that $c_{X, n N}^{4,2}$ is injective for all $n \in \mathbb{Z}_{>0}$. Moreover, we have $\operatorname{ker}\left(\psi_{X}\right)=0$.

Now we state our main result.

THEOREM 6.3. We assume the following condition:

(*) the irreducible components of $\bar{X} \backslash \bar{U}$ generate $N S(\bar{X})$.

Then we have the following:

(1) $\operatorname{ker}\left(\psi_{U}\right)=C_{0}(U)_{\text {Div }}$;

(2) $F_{0}(U):=\operatorname{ker}\left(\psi_{U} \otimes \mathbb{Q} / \mathbb{Z}\right)$ is finite, and there is an $N \in \mathbb{Z}_{>0}$ such that for all $n \in \mathbb{Z}_{>0}$ the canonical map $\operatorname{ker}\left(\psi_{U} / n N\right) \rightarrow F_{0}(U)$ is bijective;

(3) there is an injection $F_{0}(U) \hookrightarrow \operatorname{coker}\left(\phi_{X}\right)$, which is bijective if $D(C)=0$.

In Examples 6.8 and 6.10 below, we will provide concrete examples for which $F_{0}(U) \neq 0$. Theorem 1.6(1) is a consequence of this theorem and the examples. Note that Parimala and Suresh [28] have constructed smooth projective surfaces $X$ and $X^{\prime}$ such that $\operatorname{ker}\left(\psi_{X}\right) \neq C_{0}(X)_{\text {Div }}$ and $\operatorname{ker}\left(\psi_{X^{\prime}} / 2\right) \neq 0$. It seems difficult to control the cokernel of $\psi_{U}$, because, as Proposition 6.1 shows, it can easily become bigger and bigger. If we fix $X$ and vary $U$, the above theorem immediately implies the following.

Proposition 6.4. There exists an open dense subvariety $U_{0} \subset X$ such that, for any open dense subvariety $U \subset U_{0}$, the natural map $F_{0}(U) \rightarrow$ $F_{0}\left(U_{0}\right)$ is an isomorphism.

Proof. We take $U_{*} \subset X$ that satisfies (*). By Theorem 6.3(3), the map $F_{0}(U) \rightarrow F_{0}\left(U_{*}\right)$ is injective for any $U \subset U_{*}$. Since $F_{0}\left(U_{*}\right)$ is finite, there exists $U_{0} \subset U_{*}$ such that

$$
F_{0}\left(U_{0}\right)=\bigcap_{U \subset U_{*}} F_{0}(U)
$$

The proposition holds with this $U_{0}$.

As for the map $\rho_{U}$, we prove the following result. Theorem 1.6(2) follows from this.

Theorem 6.5. The map $\rho_{U} / n: C_{1}(U) / n \rightarrow \pi_{1}^{\mathrm{ab}}(U) / n$ is an isomorphism for any $n \in \mathbb{Z}_{>0}$. We have $\operatorname{ker}\left(\rho_{U}\right)=C_{1}(U)_{\operatorname{Div}}$ and $\pi_{1}^{\mathrm{ab}}(U)=\overline{\operatorname{Im}\left(\rho_{U}\right)}$. 
Note that Sato [32] has constructed smooth projective $K 3$ surfaces $X, X^{\prime}$ such that $\operatorname{ker}\left(\rho_{X}\right) \neq C_{1}(X)_{\text {Div }}$ and $\operatorname{ker}\left(\rho_{X^{\prime}} / 2 n\right) \neq 0$ for all $n \in \mathbb{Z}_{>0}$.

Before we start the proof of Theorems 6.3 and 6.5 , we introduce a few lemmas.

LEMMA 6.6. We have a canonical isomorphism

$$
H_{\mathrm{Zar}}^{0}\left(X, \mathcal{H}^{3}(\mathbb{Q} / \mathbb{Z}(2))\right) \cong \operatorname{coker}\left[\phi_{X}: A_{0}(X) \rightarrow H_{\text {Gal }}^{1}(k, S)\right] .
$$

In particular, $H_{\mathrm{Zar}}^{0}\left(X, \mathcal{H}^{3}(\mathbb{Q} / \mathbb{Z}(2))\right)$ is finite. $\mathbb{Z}_{>0}$

Proof. By the Kummer sequence, we get an exact sequence for any $n \in$

$$
0 \rightarrow S(k) / n \rightarrow H_{\text {Gal }}^{1}(k, S[n]) \rightarrow H_{\text {Gal }}^{1}(k, S)[n] \rightarrow 0 .
$$

Using the facts that $H_{\text {Gal }}^{1}(k, S)$ is a torsion group and that the HochschildSerre spectral sequence induces an isomorphism $H_{\text {ét }}^{3}(X, \mathbb{Q} / \mathbb{Z}(2)) \cong H_{\text {Gal }}^{1}(k$, $\left.S_{\text {Tor }}\right)$, we get an exact sequence which fits into the lower row in the commutative diagram

$$
\begin{array}{ccccc}
0 \rightarrow H_{M}^{3}(X, \mathbb{Z}(2)) \otimes \mathbb{Q} / \mathbb{Z} & \rightarrow & H_{M}^{3}(X, \mathbb{Q} / \mathbb{Z}(2)) & \rightarrow & \mathrm{CH}_{0}(X)_{\text {Tor }} \rightarrow 0 \\
\downarrow & \cong & \downarrow^{c_{X, \infty}^{3,2}} & & \downarrow^{\phi_{X}} \\
0 \rightarrow \quad S(k) \otimes \mathbb{Q} / \mathbb{Z} & \rightarrow & H_{\text {ét }}^{3}(X, \mathbb{Q} / \mathbb{Z}(2)) & \rightarrow & H_{\text {Gal }}^{1}(k, S) \rightarrow 0
\end{array}
$$

(Note that $\mathrm{CH}_{0}(X)_{\text {Tor }}=A_{0}(X)$.) Here the upper row is exact by (2.1.2). The left vertical isomorphism is given by [3, Theorem $\mathrm{C}]$. (Here we used the assumption that $X(k) \neq \phi$.) Now the assertion follows from Theorem 6.2 and Lemma 3.5.

Lemma 6.7. If $(*)$ is satisfied, then $H_{\text {ét }, c}^{3}(V, \mathbb{Q} / \mathbb{Z}(2)) \rightarrow H_{\text {ét, } c}^{3}(V \backslash U$, $\mathbb{Q} / \mathbb{Z}(2))$ is injective.

Proof. It suffices to show that $H_{\text {ét,c }}^{3}(U, \mathbb{Q} / \mathbb{Z}(2)) \rightarrow H_{\text {ét,c }}^{3}(V, \mathbb{Q} / \mathbb{Z}(2))$ is the zero map. By Poincaré duality, this amounts to showing that $H_{\text {ét }}^{3}(V$, $\hat{\mathbb{Z}}(1)) \rightarrow H_{\text {ét }}^{3}(U, \hat{\mathbb{Z}}(1))$ is the zero map. By the Gysin sequence $H_{\text {ét }}^{3}(X, \hat{\mathbb{Z}}(1)) \rightarrow$ $H_{\text {ét }}^{3}(V, \hat{\mathbb{Z}}(1)) \rightarrow H_{\text {ét }}^{0}(X \backslash V, \hat{\mathbb{Z}}(-1))=0$, it suffices to show that $H_{\text {ét }}^{3}(X$, $\hat{\mathbb{Z}}(1)) \rightarrow H_{\text {ét }}^{3}(U, \hat{\mathbb{Z}}(1))$ is the zero map. By the Hochschild-Serre spectral sequence, we have $H_{\text {ét }}^{3}(X, \hat{\mathbb{Z}}(1))=H_{\text {Gal }}^{1}\left(k, H_{\text {ét }}^{2}(\bar{X}, \hat{\mathbb{Z}}(1))\right)$, and we are reduced to showing that $N S(\bar{X}) \otimes \hat{\mathbb{Z}}=H_{\text {ét }}^{2}(\bar{X}, \hat{\mathbb{Z}}(1)) \rightarrow H_{\text {ét }}^{2}(\bar{U}, \hat{\mathbb{Z}}(1))$ is the zero map, but this follows from the assumption $(*)$. 
Proof of Theorem 6.3. Let $N_{1}=N_{X} \in \mathbb{Z}_{>0}$ be the natural number appearing in Theorem 6.2 , and let $N_{2}$ be the order of $H_{\mathrm{Zar}}^{0}\left(X, \mathcal{H}^{3}(\mathbb{Q} / \mathbb{Z}(2))\right)$. Put $N=N_{1} N_{2}$, and take any $n \in \mathbb{Z}_{>0}$. By Theorem 6.2 and Lemma 3.5, we have $\operatorname{coker}\left(c_{X, n N}^{3,2}\right) \cong H_{\text {Zar }}^{0}\left(X, \mathcal{H}^{3}(\mathbb{Z} / n N(2))\right)$. Hence, we have a commutative diagram which contains the map $\eta_{n}$ appearing in Proposition 3.4:

$$
\begin{array}{cccc}
H_{\text {Zar }}^{0}\left(X, \mathcal{H}^{3}(\mathbb{Z} / n N(2))\right) & \stackrel{\eta_{n N}}{\rightarrow} & \operatorname{coker}\left(c_{C, n N}^{3,2}\right) \cong(\mathbb{Z} / n N)^{\oplus r(C)} \\
\| & & \downarrow & \cap \\
H_{\text {Zar }}^{0}\left(X, \mathcal{H}^{3}(\mathbb{Q} / \mathbb{Z}(2))\right) & \stackrel{\eta_{\infty}}{\rightarrow} & \operatorname{coker}\left(c_{C, \infty}^{3,2}\right) & \cong(\mathbb{Q} / \mathbb{Z})^{\oplus r(C)}
\end{array}
$$

where the two right horizontal isomorphisms are given by Theorem 5.2. We get $\operatorname{ker}\left(\eta_{n N}\right) \cong \operatorname{ker}\left(\eta_{\infty}\right)$. Then by Theorem 6.2 and Proposition 3.4, we have a commutative diagram,

$$
\begin{array}{ccc}
\operatorname{ker}\left(\eta_{n N}\right) & \rightarrow & \operatorname{ker}\left(\psi_{U} / n N\right) \\
\downarrow & £ & \downarrow \\
\operatorname{ker}\left(\eta_{\infty}\right) & \cong & \operatorname{ker}\left(\psi_{U} \otimes \mathbb{Q} / \mathbb{Z}\right)=F_{0}(U)
\end{array}
$$

in which the top horizontal map is surjective. Hence, all the maps in this diagram are bijective. This proves (2). If $D(C)=0$, then we have $\operatorname{ker}\left(\eta_{\infty}\right)=$ $H_{\text {Zar }}^{0}\left(X, \mathcal{H}^{3}(\mathbb{Q} / \mathbb{Z}(2))\right)$, and Lemma 6.6 proves (3). Claim (1) follows from Lemma 4.5.

Proof of Theorem 6.5. By Proposition 3.4 and Lemma 4.1, it suffices to show the bijectivity of $c_{X, n}^{i, 3}$ for $i=4,5$ and $n \in \mathbb{Z}_{>0}$. Probably this is well known to the specialists, but the author could not find a suitable reference. For the sake of completeness, we include a proof here.

Let $n \in \mathbb{Z}_{>0} \cup\{\infty\}$. The injectivity of $c_{X, n}^{4,3}$ follows from Proposition 3.2. We consider the commutative diagram with exact rows

$$
\begin{aligned}
& 0 \rightarrow H_{0}^{M}(X, \mathbb{Z}(-1)) / n \quad \rightarrow H_{0}^{M}(X, \mathbb{Z} / n(-1)) \rightarrow C_{1}(X)[n] \quad \rightarrow 0
\end{aligned}
$$

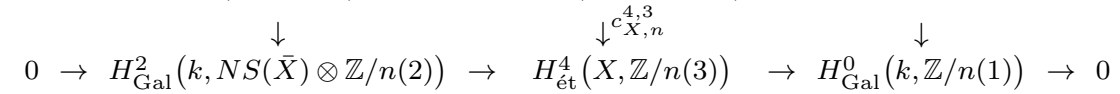

Here the upper and lower rows are given by (2.1.2) and by the HochschildSerre spectral sequence, respectively. The right vertical map is given by

$$
C_{1}(X)[n] \rightarrow C_{1}(\operatorname{Spec} k)[n]=k^{*}[n]=H_{\text {Gal }}^{0}(k, \mathbb{Z} / n(1)) .
$$


The left vertical map is defined by the commutativity of this diagram. The existence of a $k$-rational point of $X$ shows that both rows are split exact. It also shows the surjectivity of the right vertical map. We show the surjectivity of the left vertical map. Since the corestriction map

$$
H_{\text {Gal }}^{2}\left(k^{\prime}, N S(\bar{X}) \otimes \mathbb{Z} / n(2)\right) \rightarrow H_{\text {Gal }}^{2}(k, N S(\bar{X}) \otimes \mathbb{Z} / n(2))
$$

is surjective for any finite extension $k^{\prime} / k$, it suffices to show this surjectivity when $X$ is a split rational surface (i.e., $k(X)$ is purely transcendental over $k$ ). This follows from Lemma 3.5 because $H_{\text {Zar }}^{0}\left(X, \mathcal{H}^{4}(\mathbb{Z} / n(3))\right)=0$ when $X$ is a split rational surface. (More explicitly, one can directly show the surjectivity of the composite map

$$
\begin{aligned}
\operatorname{Pic}(X) \otimes K_{2} k & \stackrel{\text { prod. }}{\rightarrow} H_{M}^{4}(X, \mathbb{Z}(3)) \\
& \cong H_{0}^{M}(X, \mathbb{Z}(-1)) \rightarrow H_{\text {Gal }}^{2}(k, N S(\bar{X}) \otimes \mathbb{Z} / n(2)),
\end{aligned}
$$

where the first map is given by the product structure.)

Next, we consider $c_{X, n}^{5,3}$. By the Hochschild-Serre spectral sequence, we have an isomorphism

$$
H_{\text {ét }}^{5}(X, \mathbb{Z} / n(3)) \cong H_{\text {Gal }}^{1}(k, \mathbb{Z} / n(1)) \cong k^{*} / n .
$$

It suffices to show that the structure morphism induces an isomorphism $C_{1}(X) \rightarrow C_{1}(\operatorname{Spec} k)=k^{*}$. By the existence of a $k$-rational point of $X$, one knows that this map is split surjective. We set $V(X):=\operatorname{ker}\left[C_{1}(X) \rightarrow\right.$ $C_{1}($ Spec $\left.k)=k^{*}\right]$. Since $V\left(X^{\prime}\right)=0$ if $X^{\prime}$ is a split rational surface, the norm argument shows that $V(X)$ is a torsion group. It suffices to show that $V(X)_{\text {Tor }}=0$. This follows from a commutative diagram with exact rows

$$
\begin{aligned}
& 0 \rightarrow V(X)_{\text {Tor }} \rightarrow \quad C_{1}(X)_{\text {Tor }} \quad \stackrel{(!)}{\rightarrow} \quad \mu(k) \rightarrow 0 \\
& \uparrow \text { surj. } \quad \| \\
& H_{0}^{M}(X, \mathbb{Q} / \mathbb{Z}(-1)) \stackrel{(!)}{\rightarrow} H_{0}^{M}(\operatorname{Spec} k, \mathbb{Q} / \mathbb{Z}(1)) \\
& \downarrow^{c_{X, \infty}^{4,3}} \\
& H_{\text {ét }}^{4}(X, \mathbb{Q} / \mathbb{Z}(3)) \cong H_{\mathrm{Gal}}^{0}(k, \mathbb{Q} / \mathbb{Z}(1))
\end{aligned}
$$

Here the maps (!) are split surjective, and $c_{X, \infty}^{4,3}$ is injective by Proposition 3.2. The lower horizontal map is bijective because the first term in the 
bottom row of (6.2.1) vanishes when $n=\infty$. The middle upper vertical map is given by $(2.1 .2)$.

EXAMPLE 6.8. Let $a, b \in k^{*}$. Suppose that $p \neq 2, a \neq b$, and $\operatorname{ord}_{k}(a)=$ $\operatorname{ord}_{k}(b)=r$. We also take $d \in k^{*}$ such that $k(\sqrt{d}) / k$ is a nontrivial unramified extension. Let $X$ be a smooth projective surface with function field $K:=$ $k(x, y)[z] /\left(x^{2}-d y^{2}-z(z-a)(z-b)\right)$ (called a Châtelet surface). Let $C$ be a divisor on $X$, and put $U=X \backslash C$. We assume that the irreducible components of $\bar{C}$ generate $N S(\bar{X})$ and that $D(C)=0$. (When $X$ is minimal with respect to the fibration corresponding to the field extension $K / k(z)$, we can take $C$ to be the union of the four singular fibers and the " $\infty$-section," so that each component has genus zero (see Remark 5.3; see [7] for details).) We claim the following.

(1) If $r$ is even and $\operatorname{ord}_{k}(a-b)=r$, then $F_{0}(U) \cong \mathbb{Z} / 2 \oplus \mathbb{Z} / 2$.

(2) If $r$ is even and $\operatorname{ord}_{k}(a-b)>r$, then $F_{0}(U) \cong \mathbb{Z} / 2$.

(3) If $r$ is odd, then $F_{0}(U)=0$.

It is shown in [7] that $H_{\text {Gal }}^{1}(k, S) \cong \mathbb{Z} / 2 \oplus \mathbb{Z} / 2$. Hence, the claim follows from Theorem 6.3 and the following theorem.

THEOREM $6.9([9,(4.7)])$.

(1) If $r$ is even and $\operatorname{ord}_{k}(a-b)=r$, then $A_{0}(X)=0$.

(2) If $r$ is even and $\operatorname{ord}_{k}(a-b)>r$, then $A_{0}(X) \cong \mathbb{Z} / 2$.

(3) If $r$ is odd, then $A_{0}(X) \cong \mathbb{Z} / 2 \oplus \mathbb{Z} / 2$.

One can extend this example to the case where $k(\sqrt{d}) / k$ is a ramified extension, by using a result of Dalawat [10].

EXAMPLE 6.10. Let $a \in k^{*}$, and let $X$ be a cubic surface defined by $T_{0}^{3}+T_{1}^{3}+T_{2}^{3}+a T_{3}^{3}=0$ in $\mathbb{P}_{k}^{3}$. Let $\zeta \in \bar{k}$ be a primitive cubic root of unity. Set $r=\operatorname{ord}_{k}(a)$. Let $C$ be a divisor on $X$, and put $U=X \backslash C$. We assume that the irreducible components of $\bar{C}$ generate $N S(\bar{X})$ and that $D(C)=0$. (For example, we can take $C$ to be the union of the 27 lines on $X$; see Remark 5.3.) We claim the following.

(1) If $p \neq 3$ and $r \equiv 0 \bmod 3$, then $F_{0}(U) \cong \mathbb{Z} / 3 \oplus \mathbb{Z} / 3$.

(2) If $p \neq 3, r \neq 0 \bmod 3$, and $\zeta \notin k$, then $F_{0}(U) \cong \mathbb{Z} / 3$.

(3) Suppose that $\zeta \in k$. If $p \neq 3$, assume that $r \not \equiv 0 \bmod 3$. If $p=3$, assume that $r \equiv 1 \bmod 3$. Then $F_{0}(X)=0$.

Manin [24] has observed that $H_{\text {Gal }}^{1}(k, S) \cong \mathbb{Z} / 3 \oplus \mathbb{Z} / 3$. Hence, the claim follows from Theorem 6.3 and the following theorem. 
THEOREM 6.11 ([31, (5.1.1)]).

(1) If $p \neq 3$ and $r \equiv 0 \bmod 3$, then $A_{0}(X)=0$.

(2) If $p \neq 3, r \neq 0 \bmod 3$, and $\zeta \notin k$, then $A_{0}(X) \cong \mathbb{Z} / 3$.

(3) Suppose that $\zeta \in k$. If $p \neq 3$, assume that $r \neq \equiv 0 \bmod 3$. If $p=3$, assume that $r \equiv 1 \bmod 3$. Then $A_{0}(X) \cong \mathbb{Z} / 3 \oplus \mathbb{Z} / 3$.

Acknowledgments. The author expresses his gratitude to Professors Shuji Saito and Bruno Kahn for helpful comments. Thanks are also due to one of the referees for insightful comments.

\section{REFERENCES}

[1] S. Bloch, Algebraic K-theory and classfield theory for arithmetic surfaces, Ann. of Math. (2) 114 (1981), 229-265. MR 0632840. DOI 10.2307/1971294.

[2] - On the Chow groups of certain rational surfaces, Ann. Sci. Éc. Norm. Supér. (4) 14 (1981), 41-59. MR 0618730.

[3] J.-L. Colliot-Thélène, Hilbert's theorem 90 for $K_{2}$, with application to the Chow groups of rational surfaces, Invent. Math. 71 (1983), 1-20. MR 0688259. DOI 10. 1007/BF01393336.

[4] - "Cycles algébriques de torsion et $K$-théorie algébrique" in Arithmetic Algebraic Geometry (Trento, 1991), Lecture Notes in Math. 1553, Springer, Berlin, 1993, 1-49. MR 1338859.

[5] - L'arithmétique du groupe de Chow des zéro-cycles, J. Théor. Nombres Bordeaux 7 (1995), 51-73. MR 1413566.

[6] J.-L. Colliot-Thélène and S. Saito, Zéro-cycles sur les variétés p-adiques et groupe de Brauer, Int. Math. Res. Not. IMRN 1996, no. 4, 151-160. MR 1385140. DOI 10. 1155/S107379289600013X.

[7] J.-L. Colliot-Thélène and J.-J. Sansuc, "La descente sur les variétés rationnelles" in Journées de Géometrie Algébrique d'Angers, Juillet 1979, Sijthoff and Noordhoff, Alphen aan den Rijn, 1980, 223-237. MR 0605344.

[8] - On the Chow groups of certain rational surfaces: A sequel to a paper of S. Bloch, Duke Math. J. 48 (1981), 421-447. MR 0620258.

[9] D. E. Coray and M. A. Tsfasman, Arithmetic on singular del Pezzo surfaces, Proc. Lond. Math. Soc. (3) 57 (1988), 25-87. MR 0940430. DOI 10.1112/plms/s3-57.1.25.

[10] C. S. Dalawat, Le groupe de Chow d'une surface de Châtelet sur un corps local, Indag. Math. (N.S.) 11 (2000), 173-185. MR 1813158. DOI 10.1016/S0019-3577(00)89075-8.

[11] E. M. Friedlander and V. Voevodsky, "Bivariant cycle cohomology" in Cycles, Transfers, and Motivic Homology Theories, Ann. of Math. Stud. 143, Princeton University Press, Princeton, 2000, 138-187. MR 1764201.

[12] T. Geisser, Arithmetic homology and an integral version of Kato's conjecture, J. Reine Angew. Math. 644 (2010), 1-22. MR 2671773. DOI 10.1515/CRELLE.2010.050.

[13] - On Suslin's singular homology and cohomology, Doc. Math. 2010, Extra Vol., Andrei A. Suslin Sixtieth Birthday, 223-249. MR 2804255.

[14] T. Geisser and M. Levine, The Bloch-Kato conjecture and a theorem of SuslinVoevodsky, J. Reine Angew. Math. 530 (2001), 55-103. MR 1807268. DOI 10.1515/ crll.2001.006. 
[15] P. Gille and T. Szamuely, Central Simple Algebras and Galois Cohomology, Cambridge Stud. Adv. Math. 101. Cambridge University Press, Cambridge, 2006. MR 2266528. DOI 10.1017/CBO9780511607219.

[16] T. Hiranouchi, Class field theory for open curves over p-adic fields, Math. Z. 266 (2010), 107-113. MR 2670674. DOI 10.1007/s00209-009-0556-1.

[17] U. Jannsen, Weights in arithmetic geometry, Jpn. J. Math. 5 (2010), no. 1, 73-102. MR 2609323. DOI 10.1007/s11537-010-0947-4.

[18] U. Jannsen and S. Saito, Kato homology of arithmetic schemes and higher class field theory over local fields, Doc. Math. 2003, Extra Vol., Kazuya Kato's Fiftieth Birthday, 479-538. MR 2046606.

[19] - Bertini theorems and Lefschetz pencils over discrete valuation rings, with applications to higher class field theory, J. Algebraic Geom. 21 (2012), 683-705. MR 2957692.

[20] B. Kahn, Relatively unramified elements in cycle modules, J. K-Theory 7 (2011), 409-427. MR 2811710. DOI 10.1017/is011003002jkt147.

[21] K. Kato and S. Saito, Unramified class field theory of arithmetical surfaces, Ann. of Math. (2) 118 (1983), 241-275. MR 0717824. DOI 10.2307/2007029.

[22] S. Lichtenbaum, Duality theorems for curves over p-adic fields, Invent. Math. 7 (1969), 120-136. MR 0242831.

[23] Y. I. Manin, "Le groupe de Brauer-Grothendieck en géométrie diophantienne" in Actes du Congrès International des Mathématiciens (Nice, 1970), I, Gauthier-Villars, Paris, 1971, 401-411. MR 0427322.

[24] - Cubic Forms: Algebra, Geometry, Arithmetic, North-Holland Math. Libr. 4, North-Holland, Amsterdam, 1974. MR 0460349.

[25] A. Mattuck, Abelian varieties over p-adic ground fields, Ann. of Math (2) 62 (1955), 92-119. MR 0071116.

[26] C. Mazza, V. Voevodsky, and C. Weibel, Lecture Notes on Motivic Cohomology, Clay Math. Monogr. 2, Amer. Math. Soc., Providence, 2006. MR 2242284.

[27] A. S. Merkurjev, On the torsion in $K_{2}$ of local fields, Ann. of Math. (2) 118 (1983), 375-381. MR 0717828. DOI 10.2307/2007033.

[28] R. Parimala and V. Suresh, Zero-cycles on quadric fibrations: Finiteness theorems and the cycle map, Invent. Math. 122 (1995), 83-117. MR 1354955. DOI 10.1007/ BF01231440.

[29] S. Saito, Class field theory for curves over local fields, J. Number Theory 21 (1985), 44-80. MR 0804915. DOI 10.1016/0022-314X(85)90011-3.

[30] - On the cycle map for torsion algebraic cycles of codimension two, Invent. Math. 106 (1991), 443-460. MR 1134479. DOI 10.1007/BF01243920.

[31] S. Saito and K. Sato, Zero-cycles on varieties over -adic fields and Brauer groups, preprint, arXiv:0906.2273 [math.AG]

[32] K. Sato, Non-divisible cycles on surfaces over local fields, J. Number Theory 114 (2005), 272-297. MR 2167971. DOI 10.1016/j.jnt.2004.08.015.

[33] C. Scheiderer and J. van Hamel, Cohomology of tori over p-adic curves, Math. Ann. 326 (2003), 155-183. MR 1981617. DOI 10.1007/s00208-003-0416-y.

[34] A. Schmidt, Singular homology of arithmetic schemes, Algebra Number Theory 1 (2007), 183-222. MR 2361940. DOI 10.2140/ant.2007.1.183.

[35] A. Schmidt and M. Spieß, Singular homology and class field theory of varieties over finite fields, J. Reine Angew. Math. 527 (2000), 13-36. MR 1794016. DOI 10.1515/ crll.2000.079. 
[36] J.-P. Serre, Groupes algébriques et corps de classes, Hermann, Paris, 1959. MR 0103191.

[37] A. Suslin and S. Joukhovitski, Norm varieties, J. Pure Appl. Algebra 206 (2006), 245-276. MR 2220090. DOI 10.1016/j.jpaa.2005.12.012.

[38] A. Suslin and V. Voevodsky, Singular homology of abstract algebraic varieties, Invent. Math. 123 (1996), 61-94. MR 1376246. DOI 10.1007/BF01232367.

[39] - "Bloch-Kato conjecture and motivic cohomology with finite coefficients" in The Arithmetic and Geometry of Algebraic Cycles (Banff, 1998), NATO Sci. Ser. C Math. Phys. Sci. 548, Kluwer, Dordrecht, 2000, 117-189. MR 1744945.

[40] T. Szamuely, Sur l'application de réciprocité pour une surface fibrée en coniques définie sur un corps local, J. K-Theory 18 (1999), 173-179. MR 1711708. DOI 10. 1023/A:1007880216662.

[41] - Sur la théorie des corps de classes pour les variétés sur les corps p-adiques, J. Reine Angew. Math. 525 (2000), 183-212. MR 1780431. DOI 10.1515/crll.2000. 066 .

[42] V. Voevodsky, "Triangulated categories of motives over a field" in Cycles, Transfers, and Motivic Homology Theories, Ann. of Math. Stud. 143, Princeton University Press, Princeton, 2000, 188-238. MR 1764202.

[43] - On motivic cohomology with Z/l-coefficients, Ann. of Math. (2) 174 (2011), 401-438. MR 2811603. DOI 10.4007/annals.2011.174.1.11.

[44] C. Weibel, The norm residue isomorphism theorem, J. Topol. 2 (2009), 346-372. MR 2529300. DOI 10.1112/jtopol/jtp013.

[45] G. Wiesend, Class field theory for arithmetic schemes, Math. Z. 256 (2007), 717-729. MR 2308885. DOI 10.1007/s00209-006-0095-y.

[46] T. Yamazaki, On Chow and Brauer groups of a product of Mumford curves, Math. Ann. 333 (2005), 549-567. MR 2198799. DOI 10.1007/s00208-005-0675-x.

[47] - Class field theory for a product of curves over a local field, Math. Z. 261 (2009), 109-121. MR 2452639. DOI 10.1007/s00209-008-0315-8.

\author{
Mathematical Institute \\ Tohoku University, Aoba, Sendai 980-8578 \\ Japan \\ ytakao@math.tohoku.ac.jp
}

